Pacific

Journal of

Mathematics

\title{
PATTERSON-SULLIVAN CURRENTS, GENERIC STRETCHING FACTORS AND THE ASYMMETRIC LIPSCHITZ METRIC FOR OUTER SPACE
}

ILYA KAPOVICH AND MARTIN LUSTIG 


\title{
PATTERSON-SULLIVAN CURRENTS, GENERIC STRETCHING FACTORS AND THE ASYMMETRIC LIPSCHITZ METRIC FOR OUTER SPACE
}

\author{
ILYA KAPOVICH AND MARTIN LUSTIG
}

We quantitatively relate the Patterson-Sullivan currents and generic stretching factors for free group automorphisms to the asymmetric Lipschitz metric on outer space and to Guirardel's intersection number. Thus we show that, given $N \geq 2$ and $\varepsilon>0$, there exists a constant $c=c(N, \varepsilon)>0$ such that for any two trees $T, S \in \mathbf{c v}_{N}$ of covolume 1 and injectivity radius $\geq \varepsilon$, we have

$$
\left|\log \left\langle S, \mu_{T}\right\rangle-d_{L}(T, S)\right| \leq c,
$$

where $d_{L}$ is the asymmetric Lipschitz metric on the Culler-Vogtmann outer space, and where $\mu_{T}$ is the (appropriately normalized) Patterson-Sullivan current corresponding to $T$. As a corollary, we show there exist constants $C_{1} \geq 1$ and $C_{2} \geq 1$ (depending on $N, \varepsilon$ ) such that for any $T, S$ as above we have

$$
\frac{1}{C_{1}} \log i_{c}(T, S)-C_{2} \leq \log \left\langle S, \mu_{T}\right\rangle \leq C_{1} \log i_{c}(T, S)+C_{2},
$$

where $i_{c}$ is the combinatorial version of Guirardel's intersection number. We apply these results to the properties of generic stretching factors of free group automorphisms. In particular, we show that for any $N \geq 2$, there exists a constant $0<\rho_{N}<1$ such that for every automorphism $\varphi$ of $F_{N}=$ $F(A)$, we have

$$
0<\rho_{N} \leq \frac{\lambda_{A}(\varphi)}{\Lambda_{A}(\varphi)} \leq 1 .
$$

Here $\lambda_{A}$ is the generic stretching factor of $\varphi$ with respect to the free basis $A$ of $F_{N}$ and $\Lambda_{A}(\varphi)$ is the extremal stretching factor of $\varphi$ with respect to $A$.

Kapovich was supported by the Collaboration Grant no. 279836 (2013-1018) from the Simons Foundation and by the NSF grant DMS-1405146. Both authors acknowledge support from U.S. National Science Foundation grants DMS 1107452, 1107263, 1107367 "RNMS: GEometric structures And Representation varieties" (the GEAR Network).

MSC2010: primary 20F65; secondary 57M07, 37B99, 37D40.

Keywords: Culler-Vogtmann's outer space, Patterson-Sullivan measures, geodesic currents. 


\section{Introduction}

For an integer $N \geq 2$, the unprojectivized outer space $\mathrm{cv}_{N}$ is the set of all $\mathbb{R}$-trees equipped with a free discrete minimal isometric action of $F_{N}$, considered up to an $F_{N}$-equivariant isometry. We denote by $\mathrm{cv}_{N}^{1}$ the set of all $T \in \mathrm{cv}_{N}$ such that the metric graph $T / F_{N}$ has volume 1 . The closure $\overline{\mathrm{cv}}_{N}$ of $\mathrm{cv}_{N}$ with respect to the equivariant Gromov-Hausdorff convergence topology (or equivalently [Paulin 1989], with respect to the hyperbolic length function topology) consists of all very small minimal isometric actions of $F_{N}$ on $\mathbb{R}$-trees, again up to an $F_{N}$-equivariant isometry. There is a natural action of $\mathbb{R}_{>0}$ on $\overline{\mathrm{cv}}_{N}$ by multiplying the metric on a tree by a positive scalar. The subset $\mathrm{cv}_{N}$ of $\overline{\mathrm{cv}}_{N}$ is invariant under this action, and the quotient $\mathrm{CV}_{N}=\mathrm{cv}_{N} / \mathbb{R}_{>0}$ is the projectivized outer space, originally introduced by Culler and Vogtmann [1986]. The quotient $\overline{\mathrm{CV}}_{N}=\overline{\mathrm{cv}}_{N} / \mathbb{R}_{>0}$ is compact, and is called the Thurston compactification of $\mathrm{CV}_{N}$. All of the above spaces admit natural $\operatorname{Out}\left(F_{N}\right)$-actions. The space $\mathrm{CV}_{N}$ is naturally $\operatorname{Out}\left(F_{N}\right)$ equivariantly homeomorphic to $\mathrm{cv}_{N}^{1}$, but it is useful to remember that technically $\mathrm{cv}_{N}^{1}$ and $\mathrm{CV}_{N}$ are distinct objects.

There are three main quantitative tools for studying points of $\overline{\mathrm{cv}}_{N}$. The first is the so-called "asymmetric Lipschitz distance". If $T \in \mathrm{cv}_{N}$ and $S \in \overline{\mathrm{cv}}_{N}$, the extremal Lipschitz distortion is given by

$$
\Lambda(T, S):=\sup _{w \in F_{N} \backslash\{1\}} \frac{\|w\|_{S}}{\|w\|_{T}} .
$$

It is known (see [Francaviglia and Martino 2011] for details) that this supremum is actually a maximum, and that $\Lambda(T, S)$ is the infimum of the Lipschitz constants of all the $F_{N}$-equivariant Lipschitz maps $T \rightarrow S$. It is also known that for all $T, S \in \mathrm{cv}_{N}^{1}$, we have $\Lambda(T, S) \geq 1$, and that the equality holds if and only if $T=S$. The asymmetric Lipschitz distance is defined as $d_{L}(T, S):=\log \Lambda(T, S)$, where $T, S \in \mathrm{cv}_{N}^{1}$. Although it is usually the case that $d_{L}(T, S) \neq d_{L}(S, T)$, the asymmetric distance $d_{L}$ satisfies all the other properties of being a metric, and it is known that the topology defined by $d_{L}$ on $\mathrm{cv}_{N}^{1}$ coincides with the standard subspace topology for $\mathrm{cv}_{N}^{1} \subseteq \mathrm{cv}_{N}$. Moreover, for any $T, S \in \mathrm{cv}_{N}^{1}$, there exists an (in general nonunique) $d_{L}$-geodesic path from $T$ to $S$ in $\mathrm{cv}_{N}^{1}$, given by natural "folding lines" [loc. cit.]. The asymmetric distance $d_{L}$ is a useful tool in the study of the geometry of $\operatorname{Out}\left(F_{N}\right)$ and it has found significant recent applications; see, for example, [Algom-Kfir 2011; 2013; Algom-Kfir and Bestvina 2012; Bestvina 2011; Francaviglia and Martino 2011; 2012; Ladra et al. 2015; White 1991].

Another two important quantitative tools for studying outer space are two notions of a "geometric intersection number". The first of these was introduced by Guirardel [2005] in the general setting of groups acting by isometries on $\mathbb{R}$-trees. Guirardel's 
intersection number $i(T, S)$ (where $T, S \in \overline{\mathrm{cv}}_{N}$ ) is defined as the covolume of the "core" for the action of $F_{N}$ on $T \times S$. Guirardel's intersection number is symmetric and $\operatorname{Out}\left(F_{N}\right)$-invariant, and for $T, S \in \mathrm{cv}_{N}$, one always has $0 \leq i(T, S)<\infty$. However, for trees in $\partial \mathrm{cv}_{N}=\overline{\mathrm{cv}}_{N} \backslash \mathrm{cv}_{N}$, it is often the case that $i(T, S)=\infty$ and $i(\cdot, \cdot)$ is discontinuous when viewed as a function on $\overline{\mathrm{cv}}_{N} \times \overline{\mathrm{cv}}_{N}$. Still, Guirardel's intersection number is a highly useful tool when studying the asymptotic geometry of $\mathrm{cv}_{N}$ itself, particularly when looking at orbits of subgroups of $\operatorname{Out}\left(F_{N}\right)$ in $\mathrm{cv}_{N}^{1}$ and $\mathrm{cv}_{N}$. Examples of such applications can be found in [Behrstock et al. 2010; Clay et al. 2015; Clay and Pettet 2010; 2012b; Guirardel 2005; Horbez 2012].

The second notion of a "geometric intersection number" was introduced in [Kapovich and Lustig 2009]. There we constructed a geometric intersection form $\langle\cdot, \cdot\rangle: \overline{\mathrm{cv}}_{N} \times \operatorname{Curr}\left(F_{N}\right) \rightarrow \mathbb{R}_{\geq 0}$, where $\operatorname{Curr}\left(F_{N}\right)$ is the space of geodesic currents on $F_{N}$. See Section $2 \mathrm{C}$ below and [Kapovich 2005; 2006; Kapovich and Lustig 2007; 2009] for the more information and the background on geodesic currents. The geometric intersection form is continuous, $\operatorname{Out}\left(F_{N}\right)$-equivariant, and, importantly, it always gives a finite output; that is, for every $T \in \overline{\mathrm{cv}}_{N}$ and $\mu \in \operatorname{Curr}\left(F_{N}\right)$, one has $0 \leq\langle T, \mu\rangle<\infty$. If $T \in \overline{\mathrm{cv}}_{N}$ and $g \in F_{N} \backslash\{1\}$ then $\left\langle T, \eta_{g}\right\rangle=\|g\|_{T}$, where $\eta_{g} \in \operatorname{Curr}\left(F_{N}\right)$ is the "counting current" associated with $g$. By its very definition, $\langle\cdot, \cdot\rangle$ is an asymmetric gadget. However, its good properties, including finiteness and global continuity on $\overline{\mathrm{cv}}_{N}$, make the geometric intersection form a useful tool that has also found a number of significant applications to the study of the dynamics and geometry of $\operatorname{Out}\left(F_{N}\right)$. See, for example, [Bestvina and Feighn 2010; Bestvina and Reynolds 2012; Carette et al. 2012; Clay and Pettet 2012a; Coulbois and Hilion 2014; Coulbois et al. 2008b; Hamenstädt 2014a; 2014b; Kapovich and Lustig 2009; 2010a; 2010b; Mann and Reynolds 2013; Reynolds 2012].

For $\varepsilon \geq 0$, we denote by $\mathrm{cv}_{N, \varepsilon}^{1}$ the set of all $T \in \mathrm{cv}_{N}^{1}$ such that the length of the shortest simple closed loop in $T / F_{N}$ is at least $\varepsilon$. The set $\operatorname{cv}_{N, \varepsilon}^{1}$ is called the $\varepsilon$-thick part of $\mathrm{cv}_{N}^{1}$. Horbez [2012] showed that, for any fixed $\varepsilon>0$, if $T, S \in \mathrm{cv}_{N, \varepsilon}^{1}$, one has

$$
\frac{1}{K_{1}} \log i_{c}(T, S)-K_{2} \leq d_{L}(T, S) \leq K_{1} \log i_{c}(T, S)+K_{2}
$$

for some constants $K_{1} \geq 1, K_{2} \geq 0$ depending only on $N$ and $\varepsilon$. Here $i_{c}(T, S)$ is the combinatorial version of Guirardel's intersection number, where $i_{c}(T, S)$ is defined as the number of 2-cells in $\operatorname{Core}(T \times S) / F_{N}$, while $i(T, S)$ is defined as the sum of the areas of all the 2-cells in $\operatorname{Core}(T \times S) / F_{N}$. Thus if, for $S, T \in \mathrm{cv}_{N}^{1}$, the trees $T_{0}, S_{0} \in \mathrm{cv}_{N}$ are obtained from $T$ and $S$ by making all edges have length 1, then $i_{c}(T, S):=i\left(T_{0}, S_{0}\right)$. Also, following the usual convention, in (\$) we interpret $\log 0$ as $\log 0=0$.

In the present paper, for $T, S \in \mathrm{cv}_{N, \varepsilon}^{1}$, we relate $\Lambda(T, S)$ to a natural quantity defined in terms of $\langle\cdot, \cdot\rangle$. Via Horbez' result, this connection also relates the 
geometric intersection form $\langle\cdot, \cdot\rangle$ to Guirardel's geometric intersection number $i(\cdot, \cdot)$. Following the results of Furman [2002] in the general set-up of wordhyperbolic groups, Kapovich and Nagnibeda [2007] associated to every $T \in \mathrm{cv}_{N}$ its Patterson-Sullivan current. In general, the Patterson-Sullivan current is naturally defined only up to a multiplication by a positive scalar. Normalizing by the geometric intersection number with $T$ provides a canonical choice. Thus for a tree $T \in \mathrm{cv}_{N}$, we denote by $\mu_{T} \in \operatorname{Curr}\left(F_{N}\right)$ the Patterson-Sullivan current associated to $T$, normalized so that $\left\langle T, \mu_{T}\right\rangle=1$. We refer the reader to Section 4 below and to [Furman 2002; Kapovich and Nagnibeda 2007; 2010] for the precise definitions and background information about the Patterson-Sullivan currents. A key result obtained by Kapovich and Nagnibeda [2007] shows that the map $J_{P S}: \mathrm{cv}_{N}^{1} \rightarrow \operatorname{Curr}\left(F_{N}\right)$, $T \mapsto \mu_{T}$ is a continuous $\operatorname{Out}\left(F_{N}\right)$-equivariant embedding.

Our main result (see Theorem 4.2 below) is:

Theorem 1.1. Let $N \geq 2$ and $\varepsilon>0$. Then there exist constants $0<\delta_{1} \leq \delta_{2}$ such that for every $T \in \mathrm{cv}_{N, \varepsilon}^{1}$ and every $S \in \overline{\mathrm{cv}}_{N}$ we have

$$
\delta_{1} \leq \frac{\left\langle S, \mu_{T}\right\rangle}{\Lambda(T, S)} \leq \delta_{2} .
$$

Therefore there exists a constant $c=c(N, \varepsilon)>0$ such that for every $T \in \mathrm{cv}_{N, \varepsilon}^{1}$ and $S \in \mathrm{cv}_{N}^{1}$ we have

$$
\left|\log \left\langle S, \mu_{T}\right\rangle-d_{L}(T, S)\right| \leq c .
$$

Using the result of Horbez [2012] stated in (\$) above, Theorem 1.1 directly implies (using the notation introduced after $(\$)$ ):

Corollary 1.2. Let $N \geq 2$ and $\varepsilon>0$. There exist constants $C_{1}, C_{2} \geq 1$ such that for any $T, S \in \mathrm{cv}_{N, \varepsilon}^{1}$, we have

$$
\frac{1}{C_{1}} \log i_{c}(T, S)-C_{2} \leq \log \left\langle S, \mu_{T}\right\rangle \leq C_{1} \log i_{c}(T, S)+C_{2} .
$$

The proof of Theorem 1.1 relies on several results regarding geodesic currents, particularly one from [Kapovich and Lustig 2009] about the continuity of the already mentioned geometric intersection form on $\overline{\mathrm{cv}}_{N} \times \operatorname{Curr}\left(F_{N}\right)$, and a result from [Kapovich and Nagnibeda 2007] saying that the Patterson-Sullivan map $\mathrm{cv}_{N}^{1} \rightarrow \operatorname{Curr}\left(F_{N}\right), T \mapsto \mu_{T}$, is a continuous $\operatorname{Out}\left(F_{N}\right)$-equivariant embedding. The crucial point in the argument uses a result from [Kapovich and Lustig 2010a] characterizing the case $\langle S, v\rangle=0$, where $S \in \overline{\mathrm{cv}}_{N}$ and $v \in \operatorname{Curr}\left(F_{N}\right)$ are arbitrary. This characterization implies that every current $\mu$ with full support (such as the Patterson-Sullivan current $\mu_{T}$ for $T \in \mathrm{cv}_{N}^{1}$ ) is filling, that is, satisfies $\langle S, \mu\rangle>0$ for every $S \in \overline{\mathrm{cv}}_{N}$. Modulo the tools mentioned above, the proof of Theorem 1.1 is not difficult (although it does require an extra trick exploiting the $\operatorname{Out}\left(F_{N}\right)$-equivariant nature of certain functions and some nice properties of $d_{L}$ ). Still, Theorem 1.1 
and its applications obtained here do provide a conceptual clarification regarding the quantitative relationships between the two notions of a geometric intersection number used in the study of $\operatorname{Out}\left(F_{N}\right)$, and about their relationship to the asymmetric Lipschitz distance.

One of our main motivations for this paper has been to better understand the properties of "generic stretching factors" for free group automorphisms.

Proposition-Definition 1.3 [Kaimanovich et al. 2007]. For any free basis $A$ of $F_{N}$ and any $S \in \overline{\mathrm{cv}}_{N}$, there exists a number $\lambda_{A}(S) \geq 0$ with the following property.

For a.e. trajectory $\xi=y_{1} y_{2} \cdots y_{n} \cdots$ of the simple nonbacktracking random walk on $F_{N}$ with respect to $A$ (that is, for a "random" geodesic ray $\xi=y_{1} y_{2} \cdots y_{n} \cdots$ over $A^{ \pm 1}$ with $\left.y_{i} \in A^{ \pm 1}\right)$, we have $\left\|y_{1} y_{2} \cdots y_{n}\right\|_{A}=n+o(n)$ and

$$
\lim _{n \rightarrow \infty} \frac{\left\|y_{1} y_{2} \cdots y_{n}\right\|_{S}}{n}=\lim _{n \rightarrow \infty} \frac{\left\|y_{1} y_{2} \cdots y_{n}\right\|_{S}}{\left\|y_{1} y_{2} \cdots y_{n}\right\|_{A}}=\lambda_{A}(S) \text {. }
$$

The number $\lambda_{A}(S)$ is called [Kapovich 2006; Kaimanovich et al. 2007] the generic stretching factor of $S$ with respect to $A$.

The term "nonbacktracking" in "nonbacktracking simple random walk" refers to the fact that for this random walk, if $x, y \in A \cup A^{-1}$, the transition probability for $x$ to be followed by $y$ is equal to $1 /(2 N-1)$ if $y \neq x^{-1}$ and is equal to 0 if $y=x^{-1}$. Thus the trajectories of this random walk are semi-infinite freely reduced words over $A^{ \pm 1}$. Informally, the generic stretching factor $\lambda_{A}(S) \geq 0$ captures the distortion $\left\|y_{1} y_{2} \cdots y_{n}\right\|_{S} / n$, where $y_{1} \cdots y_{n}$ is a "random" freely reduced word of length $n$ over $A$, as $n$ tends to infinity. The existence of $\lambda_{A}(S) \geq 0$ follows from general ergodic-theoretic considerations, as observed in [Kaimanovich et al. 2007]. As noted in Remark 4.6 below, one actually has $\lambda_{A}(S)>0$ for every $S \in \overline{\mathrm{cv}}_{N}$.

Let $A$ be a free basis of $F_{N}$ and consider the Cayley tree $T_{A} \in \mathrm{cv}_{N}$, with all edges of length $1 / N$, so that $T_{A} \in \mathrm{cv}_{N}^{1}$. Thus for every $w \in F_{N}$, we have $\|w\|_{A}=N\|w\|_{T_{A}}$, where $\|w\|_{A}$ is the cyclically reduced length of $w$ over $A^{ \pm 1}$. It is known that the Patterson-Sullivan current $\mu_{T_{A}}$ is equal to the "uniform current" $v_{A}$ on $F_{N}$ corresponding to $A$. Using the interpretation of $\left\langle S, v_{A}\right\rangle$ as the "generic stretching factor" $\lambda_{A}(S)$ of $S \in \mathrm{cv}_{N}$ with respect to $A$ [Kapovich 2006], as a consequence of Theorem 1.1 we also obtain (see Theorem 4.7 below):

Corollary 1.4. Let $N \geq 2$. There exists a constant $\delta=\delta(N) \in(0,1)$ with the following property:

For any free basis $A$ of $F_{N}$ and any $S \in \overline{\mathrm{cv}}_{N}$, we have

$$
0<\delta \leq \frac{\lambda_{A}(S)}{\Lambda\left(T_{A}, S\right)} \leq \frac{1}{N}
$$

We are particularly interested in relationships between generic stretching factors and extremal stretching factors in the context of Cayley trees of $F_{N}$ and of elements 
of $\operatorname{Out}\left(F_{N}\right)$. Note that if $A$ is a free basis of $A$ then $N T_{A} \in \mathrm{cv}_{N}$ is the standard Cayley graph of $F_{N}$ with respect to $A$, where all edges have length 1 .

If $\varphi \in \operatorname{Out}\left(F_{N}\right)$ and $w \in F_{N}$, then, since $\varphi$ is an outer automorphism, it acts on the conjugacy classes of elements of $F_{N}$ (rather than on elements of $F_{N}$ ). By convention, for $\varphi \in \operatorname{Out}\left(F_{N}\right)$ and $w \in F_{N}$, if $\varphi(w)$ appears in an expression that depends only on the conjugacy class $\varphi([w])$, we will use $\varphi(w)$ to mean any representative of that conjugacy class.

Definition 1.5 (extremal and generic stretching factors of automorphisms). Let $A$ be a free basis of $F_{N}$ and let $\varphi \in \operatorname{Out}\left(F_{N}\right)$.

Define

$$
\Lambda_{A}(\varphi):=\Lambda\left(T_{A}, T_{A} \varphi\right)=\sup _{w \neq 1} \frac{\|\varphi(w)\|_{A}}{\|w\|_{A}}=e^{d_{L}\left(T_{A}, T_{A} \varphi\right)}
$$

and refer to $\Lambda_{A}(\varphi)$ as the extremal stretching factor for $\varphi$ with respect to $A$.

Also, define $\lambda_{A}(\varphi):=\lambda_{A}\left(N T_{A} \varphi\right)=N \lambda_{A}\left(T_{A} \varphi\right)$.

Thus for a.e. trajectory $\xi=y_{1} \cdots y_{n} \cdots$ of the simple nonbacktracking random walk on $F_{N}$ with respect to $A$, we have

$$
\lambda_{A}(\varphi)=\lim _{n \rightarrow \infty} \frac{\left\|\varphi\left(y_{1} y_{2} \cdots y_{n}\right)\right\|_{A}}{n}=\lim _{n \rightarrow \infty} \frac{\left\|\varphi\left(y_{1} y_{2} \cdots y_{n}\right)\right\|_{A}}{\left\|y_{1} y_{2} \cdots y_{n}\right\|_{A}} .
$$

We call $\lambda_{A}(\varphi)$ the generic stretching factor of $\varphi$ with respect to $A$.

Thus $\Lambda_{A}(\varphi)$ measures the maximal distortion $\|\varphi(w)\|_{A} /\|w\|_{A}$ as $w$ varies over all nontrivial elements of $F_{N}$, while $\lambda_{A}(\varphi)$ captures the "generic distortion" $\|\varphi(w)\|_{A} /\|w\|_{A}$, where $w$ is a "long random" freely reduced (or cyclically reduced) word over $A^{ \pm 1}$. In practice, $\Lambda_{A}(\varphi)$ is easy to compute since it is known (see, e.g., [Francaviglia and Martino 2011]) that $\Lambda_{A}(\varphi)=\max _{1 \leq\|w\| \leq 2}\left(\|\varphi(w)\|_{A} /\|w\|_{A}\right)$.

The generic stretching factors $\lambda_{A}(\varphi)$ were introduced in [Kaimanovich et al. 2007] and further studied in [Francaviglia 2009; Kapovich 2006; Kapovich and Lustig 2010a; Sharp 2010]. In particular, it is proved in [Kaimanovich et al. 2007] that for every $\varphi \in \operatorname{Out}\left(F_{N}\right)$, the number $\lambda_{A}(\varphi)$ is rational, and moreover, $2 N \lambda_{A}(\varphi) \in \mathbb{Z}[1 /(2 N-1)]$ and there exists an algorithm that, given $\varphi$, computes $\lambda_{A}(\varphi)$. The definitions directly imply that $\lambda_{A}(\varphi) \leq \Lambda_{A}(\varphi)$. However, other than this fact, the quantitative relationship between $\Lambda_{A}(\varphi)$ and $\lambda_{A}(\varphi)$ remained unclear.

Let $N \geq 2$ and $F_{N}=F\left(a_{1}, \ldots, a_{N}\right)$ with $A=\left\{a_{1}, \ldots, a_{N}\right\}$. Define

$$
\rho_{N}:=\inf _{\varphi \in \operatorname{Out}\left(F_{N}\right)} \frac{\lambda_{A}(\varphi)}{\Lambda_{A}(\varphi)} .
$$

For every $\varphi \in \operatorname{Out}\left(F_{N}\right)$, we have $T_{A}, T_{A} \varphi \in \operatorname{cv}_{N, \varepsilon}^{1}$ with $\varepsilon=1 / N$, and thus Corollary 1.4 directly implies:

Theorem 1.6. For every $N \geq 2$ we have $\rho_{N}>0$. 
Therefore for every $\varphi \in \operatorname{Out}\left(F_{N}\right)$, we have

$$
0<\rho_{N} \leq \frac{\lambda_{A}(\varphi)}{\Lambda_{A}(\varphi)} \leq 1
$$

Our proof that $\rho_{N}>0$ does not give any explicit quantitative information about $\rho_{N}$. It would be interesting to find some explicit bounds from above and below for $\rho_{N}$, and perhaps to even compute $\rho_{N}$, at least for small values of $N$. We show in Proposition 7.1 that $\lim _{N \rightarrow \infty} \rho_{N}=0$ and that $\rho_{N}=O(1 / N)$.

As another application, we obtain (see Corollary 5.3 below):

Corollary 1.7. Let $N \geq 2$ and $F_{N}=F\left(a_{1}, \ldots, a_{n}\right)$ with $A=\left\{a_{1}, \ldots, a_{N}\right\}$. There exists $D=D(N) \geq 1$ such that for every $\varphi \in \operatorname{Out}\left(F_{N}\right)$ we have

$$
\frac{1}{D} \log \lambda_{A}(\varphi) \leq \log \lambda_{A}\left(\varphi^{-1}\right) \leq D \log \lambda_{A}(\varphi) .
$$

Let $\varphi \in \operatorname{Out}\left(F_{N}\right)$. Recall that the algebraic stretching factor $\lambda(\varphi)$ is defined as

$$
\lambda(\varphi):=\sup _{w \in F_{N}, w \neq 1} \lim _{n \rightarrow \infty} \sqrt[n]{\left\|\varphi^{n}(w)\right\|_{S}},
$$

where $S \in \mathrm{cv}_{N}$ is an arbitrary base point. It is known that the limit in the last equality always exists, that this definition of $\lambda(\varphi)$ does not depend on the choice of $S \in \mathrm{cv}_{N}$, and that we always have $\lambda(\varphi) \geq 1$. An element $\varphi \in \operatorname{Out}\left(F_{N}\right)$ is called exponentially growing if $\lambda(\varphi)>1$, and polynomially growing if $\lambda(\varphi)=1$. Indeed, it is known (see, for example, [Levitt 2009]), that $\varphi$ is polynomially growing if and only if for every $w \in F_{N}$ and $S \in \mathrm{cv}_{N}$, the sequence $\left\|\varphi^{n}(w)\right\|_{S}$ is bounded above by a polynomial in $n$.

The algebraic stretching factor $\lambda(\varphi)$ can be read off from any relative train-track representative $f: \Gamma \rightarrow \Gamma$ of $\varphi$ as the maximum of the Perron-Frobenius eigenvalues for any of the canonical irreducible diagonal blocks of the (nonnegative) transition matrix $M(f)$.

As another application of the results of this paper, we explain how the generic stretching factor $\lambda_{A}\left(\varphi^{n}\right)$ grows in terms of $n$ for an arbitrary $\varphi \in \operatorname{Out}\left(F_{N}\right)$. Thus we obtain (see Theorem 5.6 below) the following result, which answers Problem 9.2 posed in [Kaimanovich et al. 2007]:

Theorem 1.8. Let $A$ be a free basis of $F_{N}$, let $\varphi \in \operatorname{Out}\left(F_{N}\right)$ and let $\lambda(\varphi)$ be the algebraic stretching factor of $\varphi$. Then there exist constants $c_{1}, c_{2}>0$ and an integer $m \geq 0$ such that for every $n \geq 1$, we have

$$
c_{1} \lambda(\varphi)^{n} n^{m} \leq \lambda_{A}\left(\varphi^{n}\right) \leq c_{2} \lambda(\varphi)^{n} n^{m} .
$$

Moreover, if $\varphi$ admits an expanding train-track representative with an irreducible transition matrix (e.g., if $\varphi$ is fully irreducible), then $m=0$ and $\lambda(\varphi)>1$. 
The "polynomial growth degree" $m$ in this result is bounded above by the number of strata of any relative train track representative $f$ as above which have PF-eigenvalue equal to $\lambda$, and it has been determined precisely by Levitt [2009], see the proof of Proposition 5.4 below.

\section{Preliminaries}

2A. Basic terminology and notations related to outer space. We denote by $\mathrm{cv}_{N}$ the unprojectivized outer space, that is, the space of all free discrete minimal isometric actions of $F_{N}$ on $\mathbb{R}$-trees, considered up to $F_{N}$-equivariant isometry. Denote by $\overline{\mathrm{cv}}_{N}$ the closure of $\mathrm{cv}_{N}$ in the equivariant Gromov-Hausdorff convergence topology (or, equivalently, in the hyperbolic length functions topology). It is known [Bestvina and Feighn 1993; Cohen and Lustig 1995; Guirardel 1998] that $\overline{\mathrm{cv}}_{N}$ consists of all the very small nontrivial minimal isometric actions of $F_{N}$ on $\mathbb{R}$-trees, again considered up to $F_{N}$-equivariant isometry. Recall that a point $T \in \overline{\mathrm{cv}}_{N}$ is uniquely determined by its translation length function $\|\cdot\|_{T}: F_{N} \rightarrow[0, \infty)$, where for $w \in F_{N}$, we have $\|w\|_{T}=\inf _{x \in T} d(x, w x)=\min _{x \in T} d(x, w x)$.

The space $\overline{\mathrm{cv}}_{N}$ has a natural right $\operatorname{Out}\left(F_{N}\right)$-action, where for $w \in F_{N}$ and $T \in \overline{\mathrm{cv}}_{N}$, we have $\|w\|_{T \varphi}=\|\varphi(w)\|_{T}$. It is sometimes useful to convert this action to a left $\operatorname{Out}\left(F_{N}\right)$-action by setting $\varphi T:=T \varphi^{-1}$. Define

$$
\operatorname{cv}_{N}^{1}:=\left\{T \in \mathrm{cv}_{N} \mid \operatorname{vol}\left(T / F_{N}\right)=1\right\},
$$

and refer to $\mathrm{cv}_{N}^{1}$ as the volume-normalized outer space or just normalized outer space. Then $\mathrm{cv}_{N}$ is an open dense $\operatorname{Out}\left(F_{N}\right)$-invariant subset of $\overline{\mathrm{cv}}_{N}$, and $\mathrm{cv}_{N}^{1}$ is a closed Out $\left(F_{N}\right)$-invariant subset of $\mathrm{cv}_{N}$ (but of course $\mathrm{cv}_{N}^{1}$ is not closed in $\overline{\mathrm{cv}}_{N}$ ).

There is a natural action of $\mathbb{R}_{>0}$ on $\mathrm{cv}_{N}$ and $\overline{\mathrm{cv}}_{N}$ by scalar multiplication, which yields the corresponding projectivizations $\mathrm{CV}_{N}=\mathrm{cv}_{N} / \mathbb{R}_{>0}$ and $\overline{\mathrm{CV}}_{N}=\overline{\mathrm{cv}}_{N} / \mathbb{R}_{>0}$. For a tree $T \in \overline{\mathrm{cv}}_{N}$, we denote its projective class in $\overline{\mathrm{CV}}_{N}$ by [T]. Thus $[T]=$ $\{c T \mid c>0\}$. Note that $\mathrm{CV}_{N}$ is canonically $\operatorname{Out}\left(F_{N}\right)$ equivariantly homeomorphic to $\mathrm{cv}_{N}^{1}$, but it is still important to remember that technically $\mathrm{CV}_{N}$ and $\mathrm{cv}_{N}^{1}$ are distinct objects.

For $\varepsilon>0$, we denote by $\mathrm{cv}_{N, \varepsilon}^{1}$ the set of all $T \in \mathrm{cv}_{N}^{1}$ such that the shortest nontrivial immersed circuit in the metric graph $T / F_{N}$ has length $\geq \varepsilon$. Equivalently, $\operatorname{cv}_{N, \varepsilon}^{1}$ is the set of all $T \in \operatorname{cv}_{N}^{1}$ such that for every $w \in F_{N} \backslash\{1\}$, we have $\|w\|_{T} \geq \varepsilon$. For every $\varepsilon>0$, the set $\mathrm{cv}_{N, \varepsilon}^{1} \subseteq \mathrm{cv}_{N}^{1}$ is a closed $\operatorname{Out}\left(F_{N}\right)$-invariant subspace, and the quotient $\mathrm{cv}_{N, \varepsilon}^{1} / \operatorname{Out}\left(F_{N}\right)$ is compact.

A chart on $F_{N}$ is an isomorphism $\alpha: F_{N} \rightarrow \pi_{1}(\Gamma, p)$, where $\Gamma$ is a finite connected graph with all vertices of degree $\geq 3$ and where $p$ is a base vertex in $\Gamma$ (which is usually suppressed). Every such $\alpha$ defines an open cone in $\mathrm{cv}_{N}$ consisting of assigning arbitrary positive lengths to edges of $\Gamma$ and then lifting this assignment 
to the universal cover $\widetilde{\Gamma}$ to get an element $T \in \mathrm{cv}_{N}$. The intersection of such an open cone with $\mathrm{cv}_{N}^{1}$ is an open simplex $\Delta$ in $\mathrm{cv}_{N}^{1}$ of dimension $m-1$, where $m$ is the number of nonoriented edges of $\Gamma$. Every point $T \in \mathrm{cv}_{N}$ belongs to a unique open cone of this form, and every point of $\mathrm{cv}_{N}^{1}$ belongs to a unique such open simplex $\Delta$.

The space $\overline{\mathrm{CV}}_{N}$ is known to be compact and finite-dimensional.

2B. Asymmetric Lipschitz distance. For points $T \in \mathrm{cv}_{N}$ and $S \in \overline{\mathrm{cv}}_{N}$, define

$$
\Lambda(T, S)=\sup _{w \in F_{N} \backslash\{1\}} \frac{\|w\|_{S}}{\|w\|_{T}} .
$$

If $T, S \in \mathrm{cv}_{N}^{1}$, we also define $d_{L}(T, S):=\log \Lambda(T, S)$. As noted in the Introduction, for $T, S \in \mathrm{cv}_{N}^{1}$, the quantity $d_{L}(T, S)$ is often called the asymmetric Lipschitz distance from $T$ to $S$.

Remark 2.1. If $T \in \mathrm{cv}_{N}$ and $S \in \overline{\mathrm{cv}}_{N}$ then $0<\Lambda(T, S)<\infty$. Moreover, it is known [Francaviglia and Martino 2011; White 1991] that for any open simplex $\Delta \subset \mathrm{cv}_{N}^{1}$ as in Section 2A, there exists a finite subset $C_{\Delta} \subseteq F_{N} \backslash\{1\}$ such that for every $T \in \Delta$ and every $S \in \overline{\mathrm{cv}}_{N}$, we have

$$
\Lambda(T, S)=\max _{w \in C_{\Delta}} \frac{\|w\|_{S}}{\|w\|_{T}} .
$$

The set $C_{\Delta}$ can be chosen to be contained in the subset of all elements which are represented by paths that cross at most twice over every nonoriented edge of $\Gamma=T / F_{N}$ for $T \in \Delta$.

Note also that from the definition, we see that for every $T \in \mathrm{cv}_{N}, S \in \overline{\mathrm{cv}}_{N}$ and $\varphi \in \operatorname{Out}\left(F_{N}\right)$, one has $\Lambda(T, S)=\Lambda(\varphi T, \varphi S)$.

2C. Geodesic currents. We refer the reader to [Kapovich 2006; Kapovich and Lustig 2007; 2009; 2010a] for detailed background on geodesic currents, and we only recall a few basic definitions and facts here. Let $\partial^{2} F_{N}=\partial F_{N} \times \partial F_{N} \backslash$ diag, and endow $\partial^{2} F_{N}$ with the subspace topology and with the diagonal $F_{N}$-action by translations. A geodesic current on $F_{N}$ is a positive Borel measure $\mu$ on $\partial^{2} F_{N}$ such that $\mu$ is finite on compact subsets, $F_{N}$-invariant and "flip"-invariant (where the "flip" map $\partial^{2} F_{N} \rightarrow \partial^{2} F_{N}$ interchanges the two coordinates). The space of all geodesic currents on $F_{N}$ is denoted $\operatorname{Curr}\left(F_{N}\right)$. The space $\operatorname{Curr}\left(F_{N}\right)$ comes equipped with a natural weak*-topology and a natural left $\operatorname{Out}\left(F_{N}\right)$-action by affine homeomorphisms.

Let $\alpha: F_{N} \rightarrow \pi_{1}(\Gamma, p)$ be a chart on $F_{N}$, and consider $\widetilde{\Gamma}$ with the simplicial metric, where every edge has length 1 . Then there is a natural $F_{N}$-equivariant quasiisometry (given for any point $p \in \widetilde{\Gamma}$ by the orbit map $F_{N} \rightarrow \widetilde{\Gamma}, g \mapsto g p$ ) between $F_{N}$ and $\widetilde{\Gamma}$, which induces a canonical $F_{N}$-equivariant homeomorphism between 
$\partial F_{N}$ and $\partial \widetilde{\Gamma}$. We will therefore identify $\partial F_{N}$ with $\partial \widetilde{\Gamma}$ using this homeomorphism without invoking it explicitly, whenever it is convenient.

A nondegenerate geodesic segment $\gamma$ in $\widetilde{\Gamma}$ defines a cylinder set $\mathrm{Cyl}_{\alpha}(\gamma)$ consisting of all $(X, Y) \in \partial^{2} F_{N}$ such that the geodesic from $X$ to $Y$ in $\widetilde{\Gamma}$ passes through $\gamma$ (in the correct direction). The sets $\mathrm{Cyl}_{\alpha}(\gamma)$, as $\gamma$ varies among all nondegenerate geodesic edge-paths in $\widetilde{\Gamma}$, are compact and open, and form a basis for the topology on $\partial^{2} F_{N}$. Note that for $w \in F_{N}$, we have $\mathrm{Cyl}_{\alpha}(w \gamma)=w \operatorname{Cyl}_{\alpha}(\gamma)$. If $\mu \in \operatorname{Curr}\left(F_{N}\right)$ and $v$ is a nondegenerate reduced edge-path in $\Gamma$, we define the weight $\langle v, \mu\rangle_{\alpha}:=\mu\left(\mathrm{Cyl}_{\alpha}(\gamma)\right)$, where $\gamma$ is any lift of $v$. Since the measure $\mu$ is $F_{N}$-invariant, this definition does not depend on the specific choice of the lift $\gamma$ of $v$ to $\widetilde{\Gamma}$. A current $\mu$ is uniquely determined by its collection of weights with respect to a given chart. Moreover, if $\mu_{n}, \mu \in \operatorname{Curr}\left(F_{N}\right)$ and $\alpha$ is a chart as above, then $\lim _{n \rightarrow \infty} \mu_{n}=\mu$ in $\operatorname{Curr}\left(F_{N}\right)$ if and only if for every nondegenerate reduced edge-path $v$ in $\Gamma$, we have $\lim _{n \rightarrow \infty}\left\langle v, \mu_{n}\right\rangle_{\alpha}=\langle v, \mu\rangle_{\alpha}$.

For every $w \in F_{N} \backslash\{1\}$, there is an associated counting current $\eta_{w} \in \operatorname{Curr}\left(F_{N}\right)$, which depends only on the conjugacy class [w] of $w$ in $F_{N}$ and satisfies $\eta_{w^{-1}}=$ $\eta_{w}$ and $\eta_{w^{n}}=n \eta_{w}$ for all integers $n \geq 1$, and such that $\varphi \eta_{w}=\eta_{\varphi(w)}$ for all $\varphi \in \operatorname{Out}\left(F_{N}\right), w \in F_{N} \backslash\{1\}$. The precise definition of $\eta_{w}$ is not important at the moment, but we will recall some of its basic properties later, as necessary. The set $\left\{c \eta_{w} \mid c>0, w \in F_{N}, w \neq 1\right\}$ of the so-called rational currents is dense in $\operatorname{Curr}\left(F_{N}\right)$.

Be aware that, in general, for a representative (even a train-track representative) $f: \Gamma \rightarrow \Gamma$ of $\varphi$, one has $\langle v, \varphi \mu\rangle_{\alpha} \neq\langle[f(v)], \mu\rangle_{\alpha}$, where $[f(v)]$ denotes the edgepath obtained from $f(v)$ by reduction (that is, the iterative contraction of any backtracking path).

2D. Intersection form. Kapovich and Lustig [2009] proved the existence of a continuous geometric intersection form between points of $\overline{\mathrm{cv}}_{N}$ and geodesic currents:

Proposition 2.2 [Kapovich and Lustig 2009]. There exists a unique continuous function $\langle\cdot, \cdot\rangle: \overline{\mathrm{cv}}_{N} \times \operatorname{Curr}\left(F_{N}\right) \rightarrow[0, \infty)$, called the geometric intersection form, with the following properties:

(1) For any $\mu_{1}, \mu_{2} \in \operatorname{Curr}\left(F_{N}\right), T \in \overline{\mathrm{cv}}_{N}, c_{1}, c_{2} \geq 0$ and $r>0$, we have

$$
\left\langle r T, c_{1} \mu_{1}+c_{2} \mu_{2}\right\rangle=r c_{1}\left\langle T, \mu_{1}\right\rangle+r c_{2}\left\langle T, \mu_{2}\right\rangle .
$$

(2) For any $T \in \overline{\operatorname{cv}}_{N}, \mu \in \operatorname{Curr}\left(F_{N}\right)$ and $\varphi \in \operatorname{Out}\left(F_{N}\right)$, we have

$$
\langle\varphi T, \varphi \mu\rangle=\langle T, \mu\rangle .
$$

(3) For any $T \in \overline{\mathrm{cv}}_{N}$ and $w \in F_{N} \backslash\{1\}$, we have

$$
\left\langle T, \eta_{w}\right\rangle=\|w\|_{T} .
$$


(4) For any $T \in \mathrm{cv}_{N}$ (with the associated chart $\alpha: F_{N} \rightarrow \pi_{1}\left(T / F_{N}\right)$ ) and any $\mu \in \operatorname{Curr}\left(F_{N}\right)$, we have

$$
\langle T, \mu\rangle=\sum_{e \in \operatorname{Edges}\left(T / F_{N}\right)} \frac{1}{2}\langle e ; \mu\rangle_{\alpha},
$$

where the summation is taken over all oriented edges of the graph $T / F_{N}$.

\section{Tree-current morphisms and extremal Lipschitz distortion}

Recall that a current $\mu \in \operatorname{Curr}\left(F_{N}\right)$ is called filling if for every $S \in \overline{\mathrm{cv}}_{N}$, we have $\langle S, \mu\rangle>0$.

We proved in [Kapovich and Lustig 2010a] that for a current $\mu \in \operatorname{Curr}\left(F_{N}\right)$ and a tree $T \in \overline{\mathrm{cv}}_{N}$, we have $\langle T, \mu\rangle=0$ if and only if the support of $\mu$ is contained in the "dual algebraic lamination" of $T$ (in the sense of [Coulbois et al. 2008a]). Using this fact, it was shown in [Kapovich and Lustig 2010a] that if $\mu$ is a current with full support, then $\mu$ is filling. We denote by $\operatorname{Curr}_{\text {fill }}\left(F_{N}\right)$ the set of all filling $\mu \in \operatorname{Curr}\left(F_{N}\right)$, and endow $\operatorname{Curr}_{\text {fill }}\left(F_{N}\right)$ with the subspace topology given by the inclusion $\operatorname{Curr}_{\text {fill }}\left(F_{N}\right) \subseteq \operatorname{Curr}\left(F_{N}\right)$.

Definition 3.1 (tree-current morphism). A tree-current morphism is a continuous function $J: \operatorname{cv}_{N}^{1} \rightarrow \operatorname{Curr}\left(F_{N}\right)$ such that for every $T \in \operatorname{cv}_{N}^{1}$ and every $\varphi \in \operatorname{Out}\left(F_{N}\right)$, we have $J(\varphi T)=\varphi J(T)$.

A filling tree-current morphism is a tree-current morphism $J: \mathrm{cv}_{N}^{1} \rightarrow \operatorname{Curr}\left(F_{N}\right)$ such that for every $T \in \mathrm{cv}_{N}^{1}$, the current $J(T) \in \operatorname{Curr}\left(F_{N}\right)$ is filling.

Lemma 3.2. The function $\mathrm{cv}_{N}^{1} \times \overline{\mathrm{cv}}_{N} \rightarrow \mathbb{R},(T, S) \mapsto \Lambda(T, S)$, is continuous.

Proof. Let $T \in \mathrm{cv}_{N}^{1}$ be arbitrary.

Let $\Delta_{1}, \ldots, \Delta_{m}$ be all the open simplices in $\mathrm{cv}_{N}^{1}$ whose closures in $\mathrm{cv}_{N}^{1}$ contain $T$.

Set $C_{T}=\bigcup_{i=1}^{m} C_{\Delta_{i}}$. Note that $U=\Delta_{1} \cup \cdots \cup \Delta_{m}$ is a neighborhood of $T \operatorname{in} \mathrm{cv}_{N}^{1}$. Thus for every $T^{\prime} \in U$ and every $S \in \overline{\mathrm{cv}}_{N}$, we have

$$
\Lambda\left(T^{\prime}, S\right)=\max _{w \in C_{T}} \frac{\|w\|_{S}}{\|w\|_{T^{\prime}}} .
$$

Therefore the function $\Lambda\left(T^{\prime}, S\right)$ is continuous on $U \times \overline{\mathrm{cv}}_{N}$. Since $T \in \mathrm{cv}_{N}^{1}$ was arbitrary, the conclusion of the lemma follows.

Let $J$ be a filling tree-current morphism. Then for any $S \in \overline{\mathrm{cv}}_{N}$ and $c>0$, we have

$$
\frac{\langle S, J(T)\rangle}{\Lambda(T, S)}=\frac{\langle c S, J(T)\rangle}{\Lambda(T, c S)} .
$$


Also, since $J(T)$ is a filling current, for every $S \in \overline{\mathrm{cv}}_{N}$, we have $\langle S, J(T)\rangle>0$. Therefore we have a well-defined function

$$
f: \operatorname{cv}_{N}^{1} \times \overline{\mathrm{CV}}_{N} \rightarrow(0, \infty)
$$

given by $f(T,[S])=\langle S, J(T)\rangle / \Lambda(T, S)$, where $T \in \mathrm{cv}_{N}^{1}$ and $S \in \overline{\mathrm{cv}}_{N}$.

Lemma 3.3. Let $J$ be a filling tree-current morphism. Then the function

$$
f: \operatorname{cv}_{N}^{1} \times \overline{\mathrm{CV}}_{N} \rightarrow(0, \infty), \quad(T, S) \mapsto \frac{\langle S, J(T)\rangle}{\Lambda(T, S)}
$$

is continuous.

Proof. The conclusion of the lemma follows directly from Lemma 3.2 together with the continuity of the geometric intersection form $\langle\cdot, \cdot\rangle$.

Corollary 3.4. Let $K \subseteq \mathrm{cv}_{N}^{1}$ be a compact subset, and let $J: \mathrm{cv}_{N}^{1} \rightarrow \operatorname{Curr}_{\text {fill }}\left(F_{N}\right)$ be a filling tree-current morphism.

Then there exist $\delta_{1}=\delta_{1}(K, J)>0$ and $\delta_{2}=\delta_{2}(K, J)>0$ such that for every $T \in K$ and every $S \in \overline{\mathrm{cv}}_{N}$, we have $\delta_{1} \leq f(K,[S]) \leq \delta_{2}$.

Proof. The set $K \times \overline{\mathrm{CV}}_{N}$ is a compact Hausdorff space and, by Lemma 3.3, $f: K \times \overline{\mathrm{CV}}_{N} \rightarrow(0, \infty)$ is a continuous function. Therefore $f$ achieves a positive minimum $\delta_{1}$ and a positive maximum $\delta_{2}$ on $K \times \overline{\mathrm{CV}}_{N}$, and the conclusion of the corollary follows.

Corollary 3.5. Let $K \subseteq \mathrm{cv}_{N}^{1}$ be a compact subset, let $\mathcal{T}_{K}=\bigcup_{\varphi \in \operatorname{Out}\left(F_{N}\right)} \varphi K$ and let $J: \mathrm{cv}_{N}^{1} \rightarrow \operatorname{Curr}\left(F_{N}\right)$ be a filling tree-current morphism.

Furthermore, let $\delta_{1}=\delta_{1}(K, J)>0$ and $\delta_{2}=\delta_{2}(K, J)>0$ be the constants provided by Corollary 3.4.

Then for every $T \in \mathcal{T}_{K}$ and every $[S] \in \overline{\mathrm{CV}}_{N}$, we have

$$
0<\delta_{1} \leq \frac{\langle S, J(T)\rangle}{\Lambda(T, S)} \leq \delta_{2}<\infty
$$

Proof. Let $T \in \mathcal{T}_{K}$ and $[S] \in \overline{\mathrm{CV}}_{N}$ be arbitrary.

Then there exist $T^{\prime} \in K$ and $\varphi \in \operatorname{Out}\left(F_{N}\right)$ such that $T=\varphi T^{\prime}$. By $\varphi$-equivariance of $J$, we have $J(T)=\varphi J\left(T^{\prime}\right)$. Define $S^{\prime}=\varphi^{-1} S$, so that $\varphi S^{\prime}=S$. Then

$$
\frac{\langle S, J(T)\rangle}{\Lambda(T, S)}=\frac{\left\langle\varphi S^{\prime}, \varphi J\left(T^{\prime}\right)\right\rangle}{\Lambda\left(\varphi T^{\prime}, \varphi S^{\prime}\right)}=\frac{\left\langle S^{\prime}, J\left(T^{\prime}\right)\right\rangle}{\Lambda\left(T^{\prime}, S^{\prime}\right)}=f\left(T^{\prime},\left[S^{\prime}\right]\right) \in\left[\delta_{1} . \delta_{2}\right],
$$

where the last inclusion holds by Corollary 3.4 since $T^{\prime} \in K$.

Note that Corollary 3.5 does not require the tree-current morphism $J: \mathrm{cv}_{N}^{1} \rightarrow$ $\operatorname{Curr}_{\text {fill }}\left(F_{N}\right)$ to be injective, although in the specific applications of interest to us $J$ will be injective. 


\section{Patterson-Sullivan currents and extremal Lipschitz distortion}

4A. Volume entropy and the Patterson-Sullivan currents. We only give here a brief summary of basic definitions and facts regarding Patterson-Sullivan currents for points of $\mathrm{cv}_{N}$. We refer the reader to [Furman 2002; Coornaert 1993; Kaimanovich 1991; Kapovich and Nagnibeda 2007] for more detailed background information about Patterson-Sullivan measures and Patterson-Sullivan currents in the context of word-hyperbolic groups and Gromov-hyperbolic spaces.

Let $T \in \mathrm{cv}_{N}$, where $N \geq 2$. Since $F_{N}$ and $T$ are $F_{N}$-equivariantly quasiisometric, there is a natural identification of $\partial F_{N}$ and $\partial T$, which we will use later on.

The volume entropy $h(T)$ of $T$ is defined as

$$
h(T):=\lim _{R \rightarrow \infty} \frac{\log \left(\#\left\{w \in F_{N} \mid d_{T}(p, w p) \leq R\right\}\right)}{R},
$$

where $p \in T$ is an arbitrary base point. It is known that the above definition does not depend on the choice of a base-point $p \in T$ and that we have $h(T)>0$ for every $T \in \mathrm{cv}_{N}$. It is also known that $h(T)$ is exactly the critical exponent of the Poincaré series

$$
\Pi_{p}(s)=\sum_{w \in F_{N}} e^{-s d_{T}(p, w p)}
$$

In other words, $\Pi_{p}(s)$ converges for all $s>h(T)$ and diverges for all $s \leq h(T)$. It is also known that as $s \rightarrow h+$, any weak limit $v$ of the measures

$$
\frac{1}{\Pi_{p}(s)} \sum_{w \in F_{N}} e^{-s d_{T}(p, w p)} \operatorname{Dirac}(w p)
$$

is a probability measure supported on $\partial T=\partial F_{N}$. Any such $v$ is called a PattersonSullivan measure on $\partial F_{N}$ corresponding to $T$, and the measure class of $v$ is canonically determined by $T$. As follows from general results of Furman [2002], in this case there exists a unique, up to a scalar multiple, geodesic current $\mu$ in the measure class of $v \times v$ on $\partial^{2} F_{N}$. We call the unique scalar multiple $\mu_{T}$ of $\mu$ such that $\left\langle T, \mu_{T}\right\rangle=1$, the Patterson-Sullivan current for $T \in \mathrm{cv}_{N}$. One also has that the current $\mu_{T}$ has full support (this follows, for example, both from the general results of Furman [2002] and from the explicit formulas for $\mu_{T}$ obtained in [Kapovich and Nagnibeda 2007]).

Proposition 4.1. The map

$$
J_{P S}: \operatorname{cv}_{N}^{1} \rightarrow \operatorname{Curr}\left(F_{N}\right), T \mapsto \mu_{T}
$$

is a filling tree-current morphism. 
Proof. Since $\mu_{T}$ has full support, by a result of Kapovich and Lustig [2010a, Corollary 1.3], it follows that $\mu_{T} \in \operatorname{Curr}_{\text {fill }}\left(F_{N}\right)$. The fact that $J_{P S}$ is a continuous $\operatorname{Out}\left(F_{N}\right)$-equivariant map was proved by Kapovich and Nagnibeda [2007]. Thus $J_{P S}$ is indeed a filling tree-current morphism, as claimed.

The fact that for $T \in \mathrm{cv}_{N}^{1}$, the Patterson-Sullivan current $\mu_{T}$ is filling, i.e., that $\left\langle S, \mu_{T}\right\rangle \neq 0$ for every $S \in \overline{\mathrm{cv}}_{N}$, is quite nontrivial and does not follow directly from Proposition 2.2. This fact, which requires a general result from [Kapovich and Lustig 2010a] characterizing the case where $\langle S, \mu\rangle=0$ (where $S \in \overline{\mathrm{cv}}_{N}$ and $\left.\mu \in \operatorname{Curr}\left(F_{N}\right)\right)$, is, in a sense, the place where the real "magic" in the proofs of the main results of the present paper happens.

We now obtain Theorem 1.1 from the Introduction:

Theorem 4.2. Let $N \geq 2$ and $\varepsilon>0$. Then there exist constants $\delta_{2} \geq \delta_{2}>0$ such that for every $T \in \mathrm{cv}_{N, \varepsilon}^{1}, S \in \overline{\mathrm{cv}}_{N}$ we have

$$
\delta_{1} \leq \frac{\left\langle S, \mu_{T}\right\rangle}{\Lambda(T, S)} \leq \delta_{2} .
$$

Therefore there exists a constant $c>0$ such that for every $T \in \mathrm{cv}_{N, \varepsilon}^{1}$ and $S \in \mathrm{cv}_{N}^{1}$, we have

$$
\left|\log \left\langle S, \mu_{T}\right\rangle-d_{L}(T, S)\right| \leq c .
$$

Proof. Since $\mathrm{cv}_{N, \varepsilon}^{1} / \operatorname{Out}\left(F_{N}\right)$ is compact and the action of $\operatorname{Out}\left(F_{N}\right)$ on $\mathrm{cv}_{N, \varepsilon}^{1}$ is properly discontinuous, there exists a compact subset $K \subseteq \mathrm{cv}_{N, \varepsilon}^{1}$ such that

$$
\mathrm{cv}_{N, \varepsilon}^{1}=\mathcal{T}_{K}=\bigcup_{\varphi \in \operatorname{Out}\left(F_{N}\right)} \varphi K
$$

By Proposition 4.1, the map $J_{P S}: \operatorname{cv}_{N}^{1} \rightarrow \operatorname{Curr}\left(F_{N}\right)$ is a filling tree-current morphism. The conclusion of the theorem now follows from Corollary 3.5.

4B. Uniform currents and generic stretching factors. Kapovich and Nagnibeda also provide reasonably explicit description of $\mu_{T}$ in terms of its weights on the "cylinder subsets" of $\partial^{2} F_{N}$. The details of that description are not immediately relevant for the present paper. However, in the case where $T \in \mathrm{cv}_{N}^{1}$ and where $T / F_{N}$ is a regular metric graph (that is, a regular graph where all edges have the same length), one can give a more precise description of $\mu_{T}$ as a "uniform current" corresponding to $T$ and relate $\mu_{T}$ to the exit measure of the simple nonbacktracking random walk on $T$. We briefly recall here the description of uniform currents for the standard $N$-roses, that is for points of $\mathrm{cv}_{N}^{1}$ corresponding to free bases of $F_{N}$.

Let $A=\left\{a_{1}, \ldots, a_{N}\right\}$ be a free basis of $F_{N}$. Let $R_{N}$ be the graph given by a wedge of $N$ loop-edges $e_{1}, \ldots, e_{N}$ at a vertex $x_{0}$. By identifying $e_{i}$ with $a_{i} \in F_{N}$, we get an identification of $\alpha_{A}: F_{N} \cong \pi_{1}\left(R_{N}, x_{0}\right)$, that is, a chart on $F_{N}$. We give each edge of $R_{N}$ length $1 / N$, so that $R_{N}$ becomes a metric graph of volume 1 . 
Then the universal cover $T_{A}:=\widetilde{R}_{N}$ is an $\mathbb{R}$-tree, which can be thought of as the Cayley graph of $F_{N}$ with respect to $A$, but where all edges have length $1 / N$. The group $F_{N}$ has a natural free and discrete isometric left action on $T_{A}$ by covering transformations, with $T_{A} / F_{N}=R_{N}$. Thus $T_{A}$ is a point of $\mathrm{cv}_{N}^{1}$.

The uniform current $v_{A}$ on $F_{N}$ corresponding to $A$ is defined explicitly by its weights. Namely, for every nontrivial freely reduced word $v$ over $A^{ \pm 1}$, we have

$$
\left\langle v, v_{A}\right\rangle_{\alpha_{A}}=\frac{1}{N(2 N-1)^{|v|-1}} .
$$

One can check that this assignment of weights does define a geodesic current and that $\left\langle T_{A}, v_{A}\right\rangle=1$. Moreover, in this case we also have:

Proposition 4.3. Let $N \geq 2$ and let $A$ be a free basis of $F_{N}$. Then $\mu_{T_{A}}=v_{A}$; that is, the Patterson-Sullivan current corresponding to $T_{A}$ is exactly the uniform current $v_{A}$.

The above fact is not explicitly stated in [Kapovich and Nagnibeda 2007] but it easily follows from the explicit formulas for the weights for Patterson-Sullivan currents they obtained in the same work. Alternatively, one knows, for example, by the results of [Coornaert 1993; Lyons 1994], that for $T_{A}$ the uniform visibility measure $m_{A}$ on $\partial F_{N}=\partial T_{A}$ is a Patterson-Sullivan measure for $T_{A}$. Since $v_{A} \in$ $\operatorname{Curr}\left(F_{N}\right)$ is in the measure class of $m_{A} \times m_{A}$ and since $\left\langle T_{A}, v_{A}\right\rangle=1$, it follows from the definition of the Patterson-Sullivan current that $\mu_{T_{A}}=v_{A}$. Note that for any other $S \in \mathrm{cv}_{N}$, the intersection number $\left\langle S, v_{A}\right\rangle$ measures the distortion of a "long random geodesic" in $T_{A}$ with respect to $S$.

Recall that in the Introduction, given a free basis $A$ of $F_{N}, S \in \overline{\mathrm{cv}}_{N}$ and $\varphi \in \operatorname{Out}\left(F_{N}\right)$, we defined the generic stretching factors $\lambda_{A}(S)$ and $\lambda_{A}(\varphi)$.

Lemma 4.4. For any free basis $A$ of $F_{N}$ and any $S \in \overline{\mathrm{cv}}_{N}$, we have

$$
\lambda_{A}(S) \leq \frac{1}{N} \Lambda\left(T_{A}, S\right) .
$$

Proof. Since all edges in $T_{A}$ have length $1 / N$, for every $w \in F_{N}$, we have $\|w\|_{A}=N\|w\|_{T_{A}}$. Then for a random trajectory $\xi=y_{1} y_{2} \cdots y_{n} \cdots$ of the simple nonbacktracking random walk on $F_{N}$ with respect to $A$ we have

$$
\begin{aligned}
\lambda_{A}(S) & =\lim _{n \rightarrow \infty} \frac{\left\|y_{1} \cdots y_{n}\right\|_{S}}{\left\|y_{1} \cdots y_{n}\right\|_{A}}=\lim _{n \rightarrow \infty} \frac{\left\|y_{1} \cdots y_{n}\right\|_{S}}{N\left\|y_{1} \cdots y_{n}\right\|_{T_{A}}} \\
& =\frac{1}{N} \lim _{n \rightarrow \infty} \frac{\left\|y_{1} \cdots y_{n}\right\|_{S}}{\left\|y_{1} \cdots y_{n}\right\|_{T_{A}}} \leq \frac{1}{N} \sup _{w \neq 1} \frac{\|w\|_{S}}{\|w\|_{T_{A}}}=\frac{1}{N} \Lambda\left(T_{A}, S\right) .
\end{aligned}
$$

A key fact about generic stretching factors, originally established in [Kapovich 2006, Proposition 9.1] in slightly more limited context, is: 
Proposition 4.5. Let $A$ be a free basis of $F_{N}$ (where $N \geq 2$ ) and let $S \in \overline{\mathrm{cv}}_{N}$. Then

$$
\left\langle S, v_{A}\right\rangle=\lambda_{A}(S) \text {. }
$$

Proof. By [Kapovich 2006, Proposition 7.3], for a.e. trajectory $\xi=y_{1} y_{2} \cdots y_{n} \cdots$ of the simple nonbacktracking random walk on $F_{N}$ with respect to $A$, we have

$$
\lim _{n \rightarrow \infty} \frac{1}{n} \eta_{y_{1} \cdots y_{n}}=v_{A} \text {. }
$$

Therefore, by Proposition 2.2, for any $S \in \overline{\mathrm{cv}}_{N}$, we have

$$
\left\langle S, v_{A}\right\rangle=\lim _{n \rightarrow \infty} \frac{1}{n}\left\langle S, \eta_{y_{1} \cdots y_{n}}\right\rangle=\lim _{n \rightarrow \infty} \frac{\left\|y_{1} \cdots y_{n}\right\|_{S}}{n}=\lambda_{A}(S) .
$$

Remark 4.6. Since the current $v_{A}$ has full support and therefore $v_{A}$ is filling, Proposition 4.5 implies that for every $S \in \overline{\mathrm{cv}}_{N}$, we have $\lambda_{A}(S)>0$. (From the definition of $\lambda_{A}(S)$, one only knows that $\lambda_{A}(S) \geq 0$ and it is not a priori obvious that the case $\lambda_{A}(S)=0$ cannot occur.)

We can now obtain Corollary 1.4 from the Introduction:

Theorem 4.7. Let $N \geq 2$. Then there exists a constant $\delta=\delta(N) \in(0,1)$ with the following property:

For any free basis $A$ of $F_{N}$ and any $S \in \overline{\mathrm{cv}}_{N}$, we have

$$
0<\delta \leq \frac{\lambda_{A}(S)}{\Lambda\left(T_{A}, S\right)} \leq \frac{1}{N}
$$

Proof. Let $A$ be a free basis of $F_{N}$ and let $S \in \overline{\mathrm{cv}}_{N}$ be arbitrary. By Lemma 4.4, we have

$$
\frac{\lambda_{A}(S)}{\Lambda\left(T_{A}, S\right)} \leq \frac{1}{N}
$$

Let $\delta=\delta_{1}(\varepsilon, N)>0$ be the constant provided by Theorem 4.2. By decreasing this constant if necessary, we can always assume that $0<\delta_{1}<1$. Note that the length of the shortest essential circuit in $T_{A}$ is equal to $1 / N$.

Since $0<\varepsilon \leq 1 / N$, it follows that $T_{A} \in \mathrm{cv}_{N, \varepsilon}^{1}$. Since $\mu_{T_{A}}=v_{A}$ and $\left\langle S, v_{A}\right\rangle=$ $\lambda_{A}(S)$, by Theorem 4.2 we have

$$
0<\delta_{1} \leq \frac{\left\langle S, \mu_{T_{A}}\right\rangle}{\Lambda\left(T_{A}, S\right)}=\frac{\left\langle S, v_{A}\right\rangle}{\Lambda\left(T_{A}, S\right)}=\frac{\lambda_{A}(S)}{\Lambda\left(T_{A}, S\right)} \leq \frac{1}{N},
$$

as required.

\section{Extremal, generic and algebraic stretching factors for free group automorphisms}

We recall the notions of extremal and generic stretching factors from Definition 1.5 in the Introduction: 
Definition 5.1 (extremal and generic stretching factors of automorphisms). Let $A$ be a free basis of $F_{N}$ and let $\varphi \in \operatorname{Out}\left(F_{N}\right)$.

Define

$$
\Lambda_{A}(\varphi):=\Lambda\left(T_{A}, T_{A} \varphi\right)=\sup _{w \neq 1} \frac{\|\varphi(w)\|_{A}}{\|w\|_{A}}=e^{d_{L}\left(T_{A}, T_{A} \varphi\right)},
$$

and refer to $\Lambda_{A}(\varphi)$ as the extremal stretching factor for $\varphi$ with respect to $A$.

Also, define $\lambda_{A}(\varphi):=\lambda_{A}\left(N T_{A} \varphi\right)=N \lambda_{A}\left(T_{A} \varphi\right)$.

Thus for a.e. trajectory $\xi=y_{1} \cdots y_{n} \cdots$ of the simple nonbacktracking random walk on $F_{N}$ with respect to $A$, we have

$$
\lambda_{A}(\varphi)=\lim _{n \rightarrow \infty} \frac{\left\|\varphi\left(y_{1} y_{2} \cdots y_{n}\right)\right\|_{A}}{n}=\lim _{n \rightarrow \infty} \frac{\left\|\varphi\left(y_{1} y_{2} \cdots y_{n}\right)\right\|_{A}}{\left\|y_{1} y_{2} \cdots y_{n}\right\|_{A}} .
$$

We call $\lambda_{A}(\varphi)$ the generic stretching factor of $\varphi$ with respect to $A$.

First, we obtain, in a slightly restated form, Theorem 1.6 from the Introduction: Theorem 5.2. For every $N \geq 2$, there exists $0<\tau_{N} \leq 1$ such that if $A$ is a free basis of $F_{N}$ and $\varphi \in \operatorname{Out}\left(F_{N}\right)$ then

$$
0<\tau_{N} \leq \frac{\lambda_{A}(\varphi)}{\Lambda_{A}(\varphi)} \leq 1 .
$$

Proof. Let $A$ be a free basis of $F_{N}$. Recall that, by definition, for $\varphi \in \operatorname{Out}\left(F_{N}\right)$ we have $\lambda_{A}(\varphi)=N \lambda_{A}\left(T_{A} \varphi\right)$ and $\Lambda_{A}(\varphi)=\Lambda\left(T_{A}, T_{A} \varphi\right)$. Therefore, by Lemma 4.4, we have $\lambda_{A}(\varphi) \leq \Lambda_{A}(\varphi)$, so that $\lambda_{A}(\varphi) / \Lambda_{A}(\varphi) \leq 1$. Since for any $\varphi \in \operatorname{Out}\left(F_{N}\right)$, we have $T_{A}, T_{A} \varphi \in \mathrm{cv}_{N, \varepsilon}^{1}$ with $\varepsilon=1 / N$, the statement of the theorem now follows directly from Theorem 4.7.

For two sequences $x_{n}>0, y_{n}>0$ (where $n \geq 1$ ), we say that $x_{n}$ grows like $y_{n}$, if there exist $0<c<c^{\prime}<\infty$ such that for every $n \geq 1$, we have $c \leq x_{n} / y_{n} \leq c^{\prime}$.

We now obtain Corollary 1.7 from the Introduction:

Corollary 5.3. Let $N \geq 2$ and $F_{N}=F\left(a_{1}, \ldots, a_{n}\right)$ with $A=\left\{a_{1}, \ldots, a_{N}\right\}$. There exists $D=D(N) \geq 1$ such that for every $\varphi \in \operatorname{Out}\left(F_{N}\right)$, we have

$$
\frac{1}{D} \log \lambda_{A}(\varphi) \leq \log \lambda_{A}\left(\varphi^{-1}\right) \leq D \log \lambda_{A}(\varphi) .
$$

Proof. It follows from [Algom-Kfir and Bestvina 2012, Theorem 24] that there exists $D^{\prime}=D^{\prime}(N) \geq 1$ such that for every $\varphi \in \operatorname{Out}\left(F_{N}\right)$, we have

$$
\frac{1}{D^{\prime}} d_{L}\left(T_{A}, T_{A} \varphi\right) \leq d_{L}\left(T_{A} \varphi, T_{A}\right) \leq D^{\prime} d_{L}\left(T_{A}, T_{A} \varphi\right) .
$$

Note that $d_{L}\left(T_{A}, T_{A} \varphi\right)=\log \Lambda\left(T_{A}, T_{A} \varphi\right)=\log \Lambda_{A}(\varphi)$ and that

$$
d_{L}\left(T_{A} \varphi, T_{A}\right)=d_{L}\left(T_{A}, T_{A} \varphi^{-1}\right)=\log \Lambda\left(T_{A}, T_{A} \varphi^{-1}\right)=\log \Lambda_{A}\left(\varphi^{-1}\right) .
$$


Theorem 5.2 now implies that there exists $D^{\prime \prime}=D^{\prime \prime}(N) \geq 1$ such that for every $\varphi \in \operatorname{Out}\left(F_{N}\right)$, we have

$(* *) \quad \frac{1}{D^{\prime \prime}} \log \lambda_{A}(\varphi)-D^{\prime \prime} \leq \log \lambda_{A}\left(\varphi^{-1}\right) \leq D^{\prime \prime} \log \lambda_{A}(\varphi)+D^{\prime \prime}$.

It was proved in [Francaviglia 2009; Kapovich and Lustig 2010a] (and also follows from Theorem 5.2) that the set $\Omega_{N}:=\left\{\lambda_{A}(\varphi) \mid \varphi \in \operatorname{Out}\left(F_{N}\right)\right\}$ is a discrete subset of $[1, \infty)$. It was established in [Kaimanovich et al. 2007] that $\lambda_{A}(\varphi)=1$ if and only if $\varphi$ is a permutational automorphism with respect to $A$, that is, if and only if, after a possible composition with an inner automorphism, $\varphi$ is induced by a permutation of $A$, with possibly inverting some elements of $A$. Note that $\varphi$ is permutational with respect to $A$ if and only if $\varphi^{-1}$ is permutational with respect to $A$, so that for $\varphi \in \operatorname{Out}\left(F_{N}\right), \lambda_{A}\left(\varphi^{-1}\right)=1$ if and only if $\lambda_{A}(\varphi)=1$. It was also proved in [loc. cit.] that the minimum of $\lambda_{A}(\varphi)$, taken over all nonpermutational $\varphi$, is equal to $1+(2 N-3) /\left(2 N^{2}-N\right)$. Therefore $(* *)$ implies that there exists $D=D(N) \geq 1$ such that for every nonpermutational $\varphi \in \operatorname{Out}\left(F_{N}\right)$, we have

$$
\frac{1}{D} \log \lambda_{A}(\varphi) \leq \log \lambda_{A}\left(\varphi^{-1}\right) \leq D \log \lambda_{A}(\varphi) .
$$

If $\varphi$ is permutational, then so is $\varphi^{-1}$. In this case we have $\log \lambda_{A}\left(\varphi^{-1}\right)=$ $\log \lambda_{A}(\varphi)=0$ and $(\diamond)$ holds as well. Thus $(\diamond)$ holds for every $\varphi \in \operatorname{Out}\left(F_{N}\right)$, which completes the proof.

Recall that for $\varphi \in \operatorname{Out}\left(F_{N}\right)$, the algebraic stretching factor $\lambda(\varphi)$ is defined as

$$
\lambda(\varphi)=\sup _{w \in F_{N}, w \neq 1} \lim _{n \rightarrow \infty} \sqrt[n]{\left\|\varphi^{n}(w)\right\|_{S}}
$$

where $S \in \mathrm{cv}_{N}$ is an arbitrary base-point. As noted earlier, this definition of $\lambda(\varphi)$ does not depend on the choice of $S \in \mathrm{cv}_{N}$. The algebraic stretching factor $\lambda(\varphi)$ can be read off from any relative train-track representative $f: \Gamma \rightarrow \Gamma$ of $\varphi$ as the maximum of the Perron-Frobenius eigenvalues for any of the canonical irreducible diagonal blocks of the (nonnegative) transition matrix $M(f)$.

Corollary 5.5 below describes, given $\varphi \in \operatorname{Out}\left(F_{N}\right)$, the asymptotics of $\Lambda\left(S, S \varphi^{n}\right)$ as $n$ tends to infinity (where $S \in \overline{\mathrm{cv}}_{N}$ is an arbitrary point, the choice of which does not affect this asymptotics). The statement of Corollary 5.5 is probably known to the experts. Since the proof is not yet available in the literature, and since we need Corollary 5.5 for the applications in this paper, we include the proof here.

Proposition 5.4. Let $\varphi \in \operatorname{Out}\left(F_{N}\right)$.

(1) Let $q \geq 1$ and let $\alpha=\varphi^{q}$ admit an improved relative train-track (in the sense of [Bestvina et al. 2000]) representative $f: \Gamma \rightarrow \Gamma$. Put $\lambda:=1$ if $\alpha$ is polynomially growing (that is, if $f$ has no exponentially growing strata) and otherwise let 
$\lambda>1$ be the largest Perron-Frobenius eigenvalue of the exponentially growing strata of $f: \Gamma \rightarrow \Gamma$.

Then there exists an integer $m \geq 0$ such that for every $S \in \mathrm{cv}_{N}$, there are some constants $0<C_{1} \leq C_{2}<\infty$ such that for every $n \geq 1$,

$$
C_{1} \lambda^{n / q} n^{m} \leq \Lambda\left(S, S \varphi^{n}\right) \leq C_{2} \lambda^{n / q} n^{m}
$$

(2) If $\varphi$ admits a train-track representative $f: \Gamma \rightarrow \Gamma$ with an irreducible transition matrix and with the Perron-Frobenius eigenvalue $\lambda>1$, then for every $S \in \mathrm{cv}_{N}$, there exist $0<C_{1} \leq C_{2}<\infty$ such that for every $n \geq 1$,

$$
C_{1} \lambda^{n} \leq \Lambda\left(S, S \varphi^{n}\right) \leq C_{2} \lambda^{n}
$$

Proof. (1) Let $T \in \mathrm{cv}_{N}^{1}$ be the point corresponding to the improved relative traintrack $f: \Gamma \rightarrow \Gamma$, where all edges of $\Gamma$ are given equal length. Put $L=\{1\}$ if $f$ has no exponentially growing strata. Otherwise let $\lambda_{1} \geq \cdots \geq \lambda_{k}>1$ be all the Perron-Frobenius eigenvalues of the exponentially growing strata of $f$ and put $L=\left\{\lambda_{1}, \ldots, \lambda_{k}, 1\right\}$. Finally put $\lambda=\max L$. Thus $\lambda \geq 1$ and $\lambda=1$ if and only if $f$ has no exponential strata.

A result of Levitt [2009, Theorem 6.2] shows that there is a finite subset $M$ of $\mathbb{Z}_{\geq 0}$ such that for every nontrivial $w \in F_{N}$, there is some $\left(\lambda^{\prime}, m^{\prime}\right) \in L \times M$ such that the sequence $\left\|\alpha^{n}(w)\right\|_{T}$ grows like $\left(\lambda^{\prime}\right)^{n} n^{m^{\prime}}$. Moreover, there exists some element $1 \neq w_{0} \in F_{N}$ such that $\left\|\alpha^{n}\left(w_{0}\right)\right\|_{T}$ grows as $\lambda^{n} n^{m}$ and such that if some other $w \neq 1$ has $\left\|\alpha^{n}(w)\right\|_{T}$ growing as $\lambda^{n} n^{m^{\prime}}$ then $m^{\prime} \leq m$.

Let $D=C_{\Delta}$ be the finite subset of $F_{N}$ as in Remark 2.1, where $\Delta$ is the open simplex in $\mathrm{cv}_{N}^{1}$ containing $T$. Therefore for every $n \geq 1$, we have $\Lambda\left(T, T \varphi^{n}\right)=$ $\max _{w \in D}\left(\left\|\alpha^{n}(w)\right\|_{T} /\|w\|_{T}\right)$. Moreover, through replacing $D$ by $D \cup\left\{w_{0}\right\}$, we can assume that $w_{0} \in D$.

It follows that $\Lambda\left(T, T \alpha^{n}\right)=\max _{w \in D}\left(\left\|\alpha^{n}(w)\right\|_{T} /\|w\|_{T}\right)$ grows like $\lambda^{n} n^{m}$.

Now let $n \geq 1$ and write $n=q n_{1}+r$, where $n_{1} \geq 0$ and $0 \leq r \leq q-1$ are integers. As we have seen, $\Lambda\left(T, T \alpha^{n_{1}}\right)=\max _{w \in D}\left(\left\|\varphi^{n_{1}}(w)\right\|_{T} /\|w\|_{T}\right)$ grows like $\lambda^{n_{1}} n_{1}^{m}$. Since $0 \leq r \leq q-1$, applying $\varphi^{r}$ distorts $\|\cdot\|_{T}$ by a bounded multiplicative amount. Therefore $\Lambda\left(T, T \varphi^{n}\right)=\max _{w \in D}\left(\left\|\varphi^{n}(w)\right\|_{T} /\|w\|_{T}\right)$ grows as $\lambda^{n / q}(n / q)^{m}$, that is, as $\lambda^{n / q} n^{m}$.

Since $T$ and $S$ are $F_{N}$-equivariantly quasi-isometric, it follows that $\Lambda\left(S, S \varphi^{n}\right)=$ $\Lambda_{A}\left(\varphi^{n}\right)$ also grows like $\lambda^{n / q} n^{m}$, and the conclusion of part (1) of the proposition follows.

(2) The proof of part (2) is known (e.g., see Theorem 8.1 in [Francaviglia and Martino 2011]) and is simpler than the proof of part (1), and we leave the details to the reader. The key point is that in this case for every nontrivial $w \in F_{N}$ such that the conjugacy class of $w$ is not $\varphi$-periodic, the sequence $\left\|\varphi^{n}(w)\right\|_{S}$ grows like $\lambda^{n}$. 
Corollary 5.5. Let $\varphi \in \operatorname{Out}\left(F_{N}\right)$, let $S \in \operatorname{cv}_{N}$ and let $\lambda(\varphi)$ be the algebraic stretching factor of $\varphi$.

Then there is an integer $m \geq 0$ such that for every $S \in \mathrm{cv}_{N}$, there are some $C_{1}, C_{2}>0$ such that

$$
C_{1} \lambda(\varphi)^{n} n^{m} \leq \Lambda\left(S, S \varphi^{n}\right) \leq C_{2} \lambda(\varphi)^{n} n^{m}
$$

for all $n \geq 1$.

Proof. It is known [Bestvina et al. 2000] that some positive power $\alpha=\varphi^{q}$ of $\varphi$ admits an improved relative train track representative.

In this case we have $\lambda(\alpha)=\lambda\left(\varphi^{q}\right)=\lambda(\varphi)^{q}$, so that $[\lambda(\alpha)]^{1 / q}=\lambda(\varphi)$. The conclusion of the corollary now follows directly from part (1) of Proposition 5.4.

Now Corollary 5.5 (applied to $S=T_{A}$, which gives $\Lambda\left(S, S \varphi^{n}\right)=\Lambda_{A}\left(\varphi^{n}\right)$ ) and Theorem 5.2 directly imply Theorem 1.8 from the Introduction:

Theorem 5.6. Let $A$ be a free basis of $F_{N}$, let $\varphi \in \operatorname{Out}\left(F_{N}\right)$ and let $\lambda(\varphi)$ be the algebraic stretching factor of $\varphi$. Then there exist constants $c_{1}, c_{2}>0$ and an integer $m \geq 0$ such that for every $n \geq 1$, we have

$$
c_{1} \lambda(\varphi)^{n} n^{m} \leq \lambda_{A}\left(\varphi^{n}\right) \leq c_{2} \lambda(\varphi)^{n} n^{m} .
$$

Moreover, if $\varphi$ admits an expanding train-track representative with an irreducible transition matrix (e.g., if $\varphi$ is fully irreducible), then $m=0$ and $\lambda(\varphi)>1$.

Example 5.7. To demonstrate that the case $\lambda>1, m>0$ in Theorem 5.6 can indeed occur, we consider an example explained on p. 1138 in [Levitt 2009]. Let $N=4$ and $F_{4}=F(A)$ with $A=\left\{a_{1}, b_{1}, a_{2}, b_{2}\right\}$. Let an automorphism $\varphi: F(A) \rightarrow F(A)$ be given by

$$
\varphi\left(a_{1}\right)=a_{1} b_{1}, \quad \varphi\left(b_{1}\right)=a_{1}, \quad \varphi\left(a_{2}\right)=a_{2} b_{1} a_{1}, \quad \varphi\left(b_{2}\right)=a_{2} .
$$

For the $A$-rose $R_{A}$, the map $f: R_{A} \rightarrow R_{A}$, given by the same formula as $\varphi$, is both a global train-track and a 2-strata relative train-track representative for $\varphi$. The bottom stratum is $\left\{a_{1}, b_{1}\right\}$ and the top stratum is $\left\{a_{2}, b_{2}\right\}$. The transition matrices for both strata are the same and are equal to $B=\left(\begin{array}{ll}1 & 1 \\ 1 & 0\end{array}\right)$, which has the Perron-Frobenius eigenvalue $\lambda=(1+\sqrt{5}) / 2$. The transition matrix for $f$ has the form $M=\left(\begin{array}{ll}B & 0 \\ C & B\end{array}\right)$, where $C=\left(\begin{array}{ll}1 & 0 \\ 0 & 0\end{array}\right)$. By iterating $M$ one can see that $\left\|\varphi^{n}\left(a_{2}\right)\right\|_{A}$ grows like $n \lambda^{n}$. One can then show that in this case $\Lambda_{A}\left(\varphi^{n}\right)$ also grows as $n \lambda^{n}$. Therefore, by Theorem 5.2, $\lambda_{A}\left(\varphi^{n}\right)$ grows as $n \lambda^{n}$ as well.

\section{Other examples of filling tree-current morphisms}

The Patterson-Sullivan map $J_{P S}: \mathrm{cv}_{N}^{1} \rightarrow \operatorname{Curr}\left(F_{N}\right), T \mapsto \mu_{T}$, is just one, albeit natural and useful, example of a filling tree-current morphism. There are many other 
filling tree-current morphisms $J: \operatorname{cv}_{N}^{1} \rightarrow \operatorname{Curr}\left(F_{N}\right)$, and Corollary 3.5 is applicable to all such $J$. We indicate here some sources of such $J$, following the approach of Reiner Martin [1995]. The main idea is that if $t \mapsto \rho(t)>0$ is a monotone decreasing continuous function which approaches 0 as $t \rightarrow \infty$ "sufficiently quickly", then

$$
J_{\rho}: \operatorname{cv}_{N}^{1} \rightarrow \operatorname{Curr}\left(F_{N}\right), \quad T \mapsto \sum_{[w] \neq[1]} \rho\left(\|w\|_{T}\right) \eta_{w}
$$

is a filling tree-current morphism.

The summation here can be taken either over all nontrivial conjugacy classes $[w]$ of elements of $F_{N}$ (or over an $\operatorname{Out}\left(F_{N}\right)$-invariant set of such conjugacy classes, although in the latter case one has to take additional care to ensure that the current $J_{\rho}(T)$ is filling).

Let us first observe that such a function $J_{\rho}$ is, by its construction, always $\operatorname{Out}\left(F_{N}\right)$-equivariant: for any $T \in \mathrm{cv}_{N}^{1}$ and $\varphi \in \operatorname{Out}\left(F_{N}\right)$, we have

$$
\varphi\left(J_{\rho}(T)\right)=\sum_{[w] \neq[1]} \rho\left(\|w\|_{T}\right) \varphi\left(\eta_{w}\right)=\sum_{[w] \neq[1]} \rho\left(\|w\|_{T}\right) \eta_{\varphi(w)}
$$

and

$$
\begin{aligned}
J_{\rho}(\varphi T) & =\sum_{[w] \neq[1]} \rho\left(\|w\|_{\varphi T}\right) \eta_{w}=\sum_{[w] \neq[1]} \rho\left(\left\|\varphi^{-1}(w)\right\|_{T}\right) \eta_{w} \\
& =\sum_{\text {with } u=\varphi^{-1}(w)} \sum_{[u] \neq[1]} \rho\left(\|u\|_{T}\right) \eta_{\varphi(u)}=\varphi\left(J_{\rho}(T)\right),
\end{aligned}
$$

so that $J_{\rho}$ is indeed $\operatorname{Out}\left(F_{N}\right)$-equivariant.

We provide here a representative result of the kind described above:

Proposition 6.1. The function

$$
J: \mathrm{cv}_{N}^{1} \rightarrow \operatorname{Curr}\left(F_{N}\right), \quad T \mapsto \sum_{[w] \neq[1]} e^{-e^{\|w\|_{T}} \eta_{w},}
$$

where the sum is taken over all nontrivial root-free conjugacy classes [w] of elements of $F_{N}$, is an injective filling tree-current morphism.

Proof. Fix a free basis $A$ of $F_{N}$ and let $T_{A} \in \mathrm{cv}_{N}^{1}$ be the Cayley graph of $F_{N}$ with respect to $A$, where all edges in $T_{A}$ have length $1 / N$. For $w \in F_{N}$ denote by $\|w\|_{A}$ the cyclically reduced length of $w$ over $A^{ \pm 1}$. Thus $\|w\|_{A}=N\|w\|_{T_{A}}$. We let $R_{A}=T_{A} / F_{N}$ be the quotient metric graph, which is a wedge of $N$ loop-edges of length $1 / N$ corresponding to elements of $A$. Let $\alpha_{A}: F_{N} \rightarrow \pi_{1}\left(R_{A}\right)$ be the associated chart.

Let $T \in \mathrm{cv}_{N}^{1}$ be arbitrary and let $U$ be a compact neighborhood of $T$ in $\mathrm{cv}_{N}^{1}$. There exists a constant $C \geq 1$ such that for every $w \in F_{N}$ and every $T^{\prime} \in U$, we 
have $\|w\|_{T^{\prime}} / C \leq\|w\|_{A} \leq C\|w\|_{T^{\prime}}$. Note that for $n \geq 1$, the number of conjugacy classes $[w]$ with $\|w\|_{A} \leq n$ is at most $(2 N)^{n}$.

To show that for each $T^{\prime} \in U, J\left(T^{\prime}\right)$ is a geodesic current we only need to verify that $J\left(T^{\prime}\right)$ takes finite values on all the two-sided cylinder sets in $\partial^{2} F_{N}$ determined by the chart $\alpha_{A}$. Since every cylinder is contained in a cylinder determined by a single edge, it suffices to show that for every oriented edge $e$ of $R_{A}$, we have $\left\langle e, J\left(T^{\prime}\right)\right\rangle_{\alpha_{A}}<\infty$.

Let $T^{\prime} \in U$ and let $e$ be an edge of $R_{A}$. For every integer $n \geq 1$, set

$$
b_{n}\left(e, T^{\prime}\right):=\sum_{0.9 n \leq\|[w]\|_{A} \leq 1.1 n} e^{-e^{\|w\|_{T^{\prime}}}}\left\langle e, \eta_{w}\right\rangle_{\alpha_{A}} .
$$

Then $\left\langle e, J\left(T^{\prime}\right)\right\rangle_{\alpha_{A}} \leq \sum_{n=1}^{\infty} b_{n}\left(e, T^{\prime}\right)$. The weight $\left\langle e, \eta_{w}\right\rangle_{\alpha_{A}}$ is equal to $1 / N$ times the number of occurrences of $e^{ \pm 1}$ in the cyclically reduced circuit $\gamma_{w}$ in $R_{A}$ representing $[w]$. Hence $\left\langle e, \eta_{w}\right\rangle_{\alpha_{A}} \leq(1 / N)\|w\|_{A}$. Since $T^{\prime} \in U$, we have $\|w\|_{T^{\prime}} \geq\|w\|_{A} / C$. Hence for every $n \geq 1$ and $T^{\prime} \in U$, we have

$$
\begin{aligned}
b_{n}\left(e, T^{\prime}\right) & =\sum_{\|[w]\|_{A} \in I} e^{-e^{\|w\|_{T}}}\left\langle e, \eta_{w}\right\rangle_{\alpha_{A}} \leq \frac{1}{N} \sum_{\|[w]\|_{A} \in I} e^{-e^{\|w\|_{A} / C}\|w\|_{A}} \\
& \leq \frac{1}{N} \sum_{\|[w]\|_{A} \in I} e^{-e^{0.9 n / C}} 1.1 n \leq \frac{1.1 n}{N} e^{-e^{0.9 n / C}}(2 N)^{1.1 n} \\
& =\frac{1.1 n}{N} e^{-e^{0.9 n / C}} e^{1.1 n \log (2 N)}=\frac{1.1 n}{N} e^{1.1 n \log (2 N)-e^{0.9 n / C}},
\end{aligned}
$$

where $I=[0.9 n, 1.1 n]$. From here we see that

$$
\left\langle e, J\left(T^{\prime}\right)\right\rangle_{\alpha_{A}} \leq \sum_{n=1}^{\infty} b_{n}\left(e, T^{\prime}\right) \leq C_{1},
$$

where $C_{1}=C_{1}(U)<\infty$ is some constant depending only on $U$.

Thus for every $T^{\prime} \in U, J\left(T^{\prime}\right)$ is indeed a geodesic current on $F_{N}$, and, in particular, $J(T) \in \operatorname{Curr}\left(F_{N}\right)$.

Note that the current $J(T)$ has full support. Indeed, for every nontrivial freely reduced word $v$ over $A^{ \pm 1}$, there exists a root-free cyclically reduced word $w$ over $A^{ \pm 1}$ containing $v$ as a subword. Then $\left\langle v, \eta_{w}\right\rangle_{\alpha_{A}}>0$ and hence, from the definition of $J(T)$, we see that $\langle v, J(T)\rangle_{\alpha_{A}}>0$. Thus indeed $J(T)$ has full support and therefore, by a result of Kapovich and Lustig [2010a], the current $J(T)$ is filling.

Since an automorphism of $F_{N}$ permutes the set of all root-free nontrivial conjugacy classes in $F_{N}$, it follows from the definition of $J$ that for every $T \in \operatorname{cv}_{N}^{1}$ and every $\varphi \in \operatorname{Out}\left(F_{N}\right)$, we have $J(\varphi T)=\varphi J(T)$.

Thus we have constructed an $\operatorname{Out}\left(F_{N}\right)$-equivariant map $J: \operatorname{cv}_{N}^{1} \rightarrow \operatorname{Curr}_{\text {fill }}\left(F_{N}\right)$. 
We next observe that the map $J$ is continuous. The proof of the continuity of $J$ is similar to the proof that $J(T)$ is a current. Let $T \in \mathrm{cv}_{N}^{1}$, let $U$ be a compact neighborhood of $T$ in $\mathrm{cv}_{N}^{1}$ and let $v$ be a nontrivial freely reduced word over $A^{ \pm 1}$. Then for every $T^{\prime} \in U$, we have

$$
\left\langle v, T^{\prime}\right\rangle_{\alpha_{A}}=\sum_{[w]}\left\langle v, e^{-e^{\|w\|_{T^{\prime}}}} \eta_{w}\right\rangle_{\alpha_{A}}=\sum_{[w]} e^{-e^{\|w\|} T_{T^{\prime}}}\langle v, w\rangle_{\alpha_{A}}
$$

One can then show, by an argument similar to that used above, that there exist positive constants $M_{w}>0$ (also depending on $U$ and $v$ but independent of $\left.T^{\prime} \in U\right)$ such that for every $T^{\prime} \in U$, we have $e^{-e^{\|w\|} T^{\prime}}\langle v, w\rangle_{\alpha_{A}} \leq M_{w}$ and that $\sum_{[w]} M_{w}<\infty$. By the Weierstrass $M$-test, it follows that the series

$$
\sum_{[w]} e^{-e^{\|w\|_{T}}}\langle v, w\rangle_{\alpha_{A}}
$$

viewed as the sum of a functions on $U$, converges uniformly on $U$ and that its sum $\left\langle v, T^{\prime}\right\rangle_{\alpha_{A}}$ is a continuous function on $U$.

Since $v$ was arbitrary, the explicit description of the topology on $\operatorname{Curr}\left(F_{N}\right)$ (see [Kapovich 2006]) implies that $J$ is a continuous function on $\mathrm{cv}_{N}^{1}$, as required.

It remains to show that $J$ is injective. Fix an enumeration, without repetitions, $w_{1}, w_{2}, \ldots$ of representatives of all the nontrivial root-free conjugacy classes in $F_{N}$. Thus for every root-free nontrivial $w \in F_{N}$, there exist unique distinct $m, n \geq 1$ such that $[w]=\left[w_{m}\right]$ and $\left[w^{-1}\right]=\left[w_{n}\right]$.

For every $i \geq 1$, set $q_{i}=\left(w_{i}^{-\infty}, w_{i}^{\infty}\right) \in \partial^{2} F_{N}$ and set $Q_{i}=\left\{q_{i}\right\}$. Note that for $i, j \geq 1$, we have $\eta_{w_{j}}\left(Q_{i}\right)=1$ if $\left[w_{i}\right]=\left[w_{j}^{ \pm 1}\right]$ and $\eta_{w_{i}}\left(Q_{i}\right)=0$ otherwise. Then, by definition of $J$, for every $T \in \operatorname{cv}_{N}^{1}$ and $i \geq 1$, we have $J(T)\left(Q_{i}\right)=2 e^{-e^{\left\|w_{i}\right\|_{T}}}$. Since the function $t \mapsto 2 e^{-e^{t}}$ is strictly monotone and thus injective, it follows that knowing the current $J(T)$, we can recover $\left\|w_{i}\right\|_{T}$ for all $i \geq 1$. Hence we can recover the length function $\|\cdot\|_{T}: F_{N} \rightarrow \mathbb{R}$ and so we can also recover $T$ itself. Thus $J$ is injective, as required.

\section{Open problems}

As we have seen in Theorem 1.6, if $N \geq 2, A=\left\{a_{1}, \ldots, a_{N}\right\}$ is a fixed free basis of $F_{N}=F(A)$, then for

$$
\rho_{N}=\inf _{\varphi \in \operatorname{Out}\left(F_{N}\right)} \frac{\lambda_{A}(\varphi)}{\Lambda_{A}(\varphi)},
$$

we have $\rho_{N}>0$. In fact, one can show:

Proposition 7.1. We have $\lim _{N \rightarrow \infty} \rho_{N}=0$, and moreover, $\rho_{N}=O(1 / N)$; that $i s, \lim \sup _{N \rightarrow \infty} N \rho_{N}<\infty$. 
Proof. For $N \geq 2$ and $m \geq 1$, let $\varphi_{N, m}: F(A) \rightarrow F(A)$ be given by $\varphi_{N, m}\left(a_{1}\right)=a_{1} a_{2}^{m}$ and $\varphi_{N, m}\left(a_{i}\right)=a_{i}$ for $2 \leq i \leq N$. It is not hard to see that

$$
\Lambda_{A}\left(\varphi_{N, m}\right)=\sup _{w \neq 1} \frac{\left\|\varphi_{N, m}(w)\right\|_{A}}{\|w\|_{A}}=m+1 .
$$

For any freely reduced $w \in F(A)$, we have

$$
\left\|\varphi_{N, m}(w)\right\|_{A} \leq(m+1)\left(a_{1} ; w\right)_{A}+\sum_{i=2}^{N}\left(a_{i} ; w\right)_{A},
$$

where $\left(a_{j} ; w\right)_{A}$ is the number of occurrences of $a_{j}^{ \pm 1}$ in $w$. On the other hand, if $w_{n} \in F(A)$ is a "long random" freely reduced word of length $n$, then asymptotically we have $\left(a_{i} ; w_{n}\right)_{A} / n \stackrel{n \rightarrow \infty}{\longrightarrow} 1 / N$ for $i=1, \ldots, N$. Therefore

$$
\begin{aligned}
\lambda_{A}\left(\varphi_{N, m}\right) & \leq \lim _{n \rightarrow \infty} \frac{(m+1)\left(a_{1} ; w\right)_{A}+\sum_{i=2}^{N}\left(a_{i} ; w\right)_{A}}{n} \\
& =(m+1) \frac{1}{N}+\frac{N-1}{N}=\frac{m}{N}+1 .
\end{aligned}
$$

Hence

$$
\rho_{N} \leq \frac{\lambda_{A}\left(\varphi_{N, m}\right)}{\Lambda_{A}\left(\varphi_{N, m}\right)} \leq \frac{1+\frac{m}{N}}{m+1}
$$

By taking $m=N$, we see that $\rho_{N} \leq 2 /(N+1) \stackrel{n \rightarrow \infty}{\longrightarrow} 0$. Thus $\lim _{N \rightarrow \infty} \rho_{N}=0$ and $\lim \sup _{N \rightarrow \infty} N \rho_{N}<\infty$.

Theorem 1.6 and Proposition 7.1 naturally raise the following:

Problem 7.2. Are the values $\rho_{N}$ algorithmically computable in terms of $N$ ? What are the exact values of $\rho_{N}$ for small $N$, say for $N=2,3,4$ ? Is it true that $\rho_{N} \in \mathbb{Q}$ ? What can be said about the precise asymptotics of $\rho_{N}$ as $N \rightarrow \infty$ ? (Note that Proposition 7.1 shows that $\rho_{N}$ decays at least as fast as $1 / N$.)

Theorem 1.1 also motivates the definition of a new notion of a continuous symmetric and $\operatorname{Out}\left(F_{N}\right)$-invariant intersection number $I: \mathrm{cv}_{N}^{1} \times \mathrm{cv}_{N}^{1} \rightarrow \mathbb{R}_{>0}$, where for $T, S \in \mathrm{cv}_{N}^{1}$, we define $I(T, S):=\left\langle S, \mu_{T}\right\rangle\left\langle T, \mu_{S}\right\rangle$. The function $I(\cdot, \cdot)$ was originally suggested to us by Arnaud Hilion, as it appears to be relevant for attempting to define an analogue of the Weil-Petersson metric on $\mathrm{cv}_{N}^{1}$.

Since the Patterson-Sullivan currents are normalized so that $\left\langle T, \mu_{T}\right\rangle=1$, for $T=S$, we have $I(T, T)=1$.

Problem 7.3. (a) Is it true that for every $T, S \in \mathrm{cv}_{N}^{1}$, we have $I(T, S) \geq 1$ ?

(b) Is it true that for $T, S \in \mathrm{cv}_{N}^{1}$, we have $I(T, S)=1$ if and only if $T=S$ ?

It was shown in [Kaimanovich et al. 2007] that if $A$ is a free basis of $F_{N}$ and $\varphi \in \operatorname{Out}\left(F_{N}\right)$ then $\lambda_{A}(\varphi) \geq 1$ and that $\lambda_{A}(\varphi)=1$ if and only if $T_{A} \varphi=T_{A}$. If 
$B$ is another free basis of $F_{N}$ and $\varphi \in \operatorname{Aut}\left(F_{N}\right)$ is such that $T_{A} \varphi=T_{B}$, then $\left\langle T_{B}, \mu_{T_{A}}\right\rangle=\lambda_{A}(\varphi)$ and $\left\langle T_{A}, \mu_{T_{B}}\right\rangle=\lambda_{A}\left(\varphi^{-1}\right)$. It follows that if $A, B$ are free bases of $F_{N}$ then $I\left(T_{A}, T_{B}\right) \geq 1$ and that $I\left(T_{A}, T_{B}\right)=1$ if and only if $T_{A}=T_{B}$. However, beyond this fact nothing appears to be known about the above question.

Recently Pollicott and Sharp [2014], using a different approach, defined and studied a Weil-Petersson type metric on $\mathrm{cv}_{N}^{1}$. It would be interesting to investigate the relationship of their metric to the quantity $I(T, S)$ defined above.

\section{Acknowledgements}

We thank Matt Clay and Camille Horbez for useful discussions about Guirardel's intersection number. We are also grateful to Brian Ray and Paul Schupp for conducting helpful computer experiments with generic stretching factors of free group automorphisms.

\section{References}

[Algom-Kfir 2011] Y. Algom-Kfir, "Strongly contracting geodesics in outer space", Geom. Topol. 15:4 (2011), 2181-2233. MR 2862155 Zbl 1250.20019

[Algom-Kfir 2013] Y. Algom-Kfir, “The metric completion of outer space”, preprint, 2013. arXiv 1202. 6392

[Algom-Kfir and Bestvina 2012] Y. Algom-Kfir and M. Bestvina, "Asymmetry of outer space", Geom. Dedicata 156 (2012), 81-92. MR 2863547 Zbl 1271.20029

[Behrstock et al. 2010] J. Behrstock, M. Bestvina, and M. Clay, "Growth of intersection numbers for free group automorphisms”, J. Topol. 3:2 (2010), 280-310. MR 2011j:20072 Zbl 1209.20031

[Bestvina 2011] M. Bestvina, "A Bers-like proof of the existence of train tracks for free group automorphisms", Fund. Math. 214:1 (2011), 1-12. MR 2012m:20046 Zbl 1248.20025

[Bestvina and Feighn 1993] M. Bestvina and M. Feighn, "Outer limits”, preprint, 1993, Available at http://andromeda.rutgers.edu/feighn/papers/outer.pdf.

[Bestvina and Feighn 2010] M. Bestvina and M. Feighn, “A hyperbolic $\operatorname{Out}\left(F_{n}\right)$-complex”, Groups Geom. Dyn. 4:1 (2010), 31-58. MR 2011a:20052 Zbl 1190.20017

[Bestvina and Reynolds 2012] M. Bestvina and P. Reynolds, "The boundary of the complex of free factors", preprint, 2012. arXiv 1211.3608

[Bestvina et al. 2000] M. Bestvina, M. Feighn, and M. Handel, "The Tits alternative for $\operatorname{Out}\left(F_{n}\right)$, I: Dynamics of exponentially-growing automorphisms", Ann. of Math. (2) 151:2 (2000), 517-623. MR 2002a:20034 Zbl 0984.20025

[Carette et al. 2012] M. Carette, S. Francaviglia, I. Kapovich, and A. Martino, "Spectral rigidity of automorphic orbits in free groups", Algebr. Geom. Topol. 12:3 (2012), 1457-1486. MR 2966693 Zbl 1261.20040

[Clay and Pettet 2010] M. Clay and A. Pettet, "Twisting out fully irreducible automorphisms", Geom. Funct. Anal. 20:3 (2010), 657-689. MR 2011i:20063 Zbl 1206.20047

[Clay and Pettet 2012a] M. Clay and A. Pettet, "Current twisting and nonsingular matrices", Comment. Math. Helv. 87:2 (2012), 385-407. MR 2914853 Zbl 1286.20049 
[Clay and Pettet 2012b] M. Clay and A. Pettet, "Relative twisting in outer space", J. Topol. Anal. 4:2 (2012), 173-201. MR 2949239 Zbl 1260.57002

[Clay et al. 2015] M. Clay, J. Mangahas, and A. Pettet, "An algorithm to detect full irreducibility by bounding the volume of periodic free factors", Michigan Math. J. 64:2 (2015), 279-292. MR 3359026

[Cohen and Lustig 1995] M. M. Cohen and M. Lustig, "Very small group actions on R-trees and Dehn twist automorphisms", Topology 34:3 (1995), 575-617. MR 96g:20053 Zbl 0844.20018

[Coornaert 1993] M. Coornaert, "Mesures de Patterson-Sullivan sur le bord d'un espace hyperbolique au sens de Gromov", Pacific J. Math. 159:2 (1993), 241-270. MR 94m:57075 Zbl 0797.20029

[Coulbois and Hilion 2014] T. Coulbois and A. Hilion, "Ergodic currents dual to a real tree", Ergodic Theory and Dynamical Systems (online publication November 2014).

[Coulbois et al. 2008a] T. Coulbois, A. Hilion, and M. Lustig, " $\mathbb{R}$-trees and laminations for free groups, II: The dual lamination of an $\mathbb{R}$-tree”, J. Lond. Math. Soc. (2) 78:3 (2008), 737-754. MR 2010h:20056 Zbl 1198.20023

[Coulbois et al. 2008b] T. Coulbois, A. Hilion, and M. Lustig, " $\mathbb{R}$-trees and laminations for free groups, III: Currents and dual $\mathbb{R}$-tree metrics", J. Lond. Math. Soc. (2) 78:3 (2008), 755-766. MR 2010h:20057 Zbl 1200.20018

[Culler and Vogtmann 1986] M. Culler and K. Vogtmann, "Moduli of graphs and automorphisms of free groups”, Invent. Math. 84:1 (1986), 91-119. MR 87f:20048 Zbl 0589.20022

[Francaviglia 2009] S. Francaviglia, "Geodesic currents and length compactness for automorphisms of free groups", Trans. Amer. Math. Soc. 361:1 (2009), 161-176. MR 2009h:20044 Zbl 1166.20032

[Francaviglia and Martino 2011] S. Francaviglia and A. Martino, "Metric properties of outer space", Publ. Mat. 55:2 (2011), 433-473. MR 2012j:20128 Zbl 1268.20042

[Francaviglia and Martino 2012] S. Francaviglia and A. Martino, "The isometry group of outer space”, Adv. Math. 231:3-4 (2012), 1940-1973. MR 2964629 Zbl 06094103

[Furman 2002] A. Furman, "Coarse-geometric perspective on negatively curved manifolds and groups", pp. 149-166 in Rigidity in dynamics and geometry (Cambridge, 2000), edited by M. Burger and A. Iozzi, Springer, Berlin, 2002. MR 2003f:53062 Zbl 1064.53025

[Guirardel 1998] V. Guirardel, "Approximations of stable actions on R-trees", Comment. Math. Helv. 73:1 (1998), 89-121. MR 99e:20037 Zbl 0979.20026

[Guirardel 2005] V. Guirardel, "Cœur et nombre d'intersection pour les actions de groupes sur les arbres”, Ann. Sci. École Norm. Sup. (4) 38:6 (2005), 847-888. MR 2007e:20055 Zbl 1110.20019

[Hamenstädt 2014a] U. Hamenstädt, "The boundary of the free splitting graph and the free factor graph", preprint, 2014. arXiv 1211.1630

[Hamenstädt 2014b] U. Hamenstädt, "Lines of minima in outer space”, Duke Math. J. 163:4 (2014), 733-776. MR 3178431 Zbl 06288360

[Horbez 2012] C. Horbez, "Sphere paths in outer space”, Algebr. Geom. Topol. 12:4 (2012), 24932517. MR 3020214 Zbl 1262.57005

[Kaimanovich 1991] V. A. Kaimanovich, "Bowen-Margulis and Patterson measures on negatively curved compact manifolds", pp. 223-232 in Dynamical systems and related topics (Nagoya, 1990), edited by K. Shiraiwa, Adv. Ser. Dynam. Systems 9, World Sci. Publ., River Edge, NJ, 1991. MR 93h:58118

[Kaimanovich et al. 2007] V. Kaimanovich, I. Kapovich, and P. Schupp, "The subadditive ergodic theorem and generic stretching factors for free group automorphisms", Israel J. Math. 157 (2007), 1-46. MR 2009d:20099 Zbl 1173.20031 
[Kapovich 2005] I. Kapovich, "The frequency space of a free group", Internat. J. Algebra Comput. 15:5-6 (2005), 939-969. MR 2007a:20038 Zbl 1110.20031

[Kapovich 2006] I. Kapovich, "Currents on free groups", pp. 149-176 in Topological and asymptotic aspects of group theory, edited by R. Grigorchuk et al., Contemp. Math. 394, Amer. Math. Soc., Providence, RI, 2006. MR 2007k:20094 Zbl 1110.20034

[Kapovich and Lustig 2007] I. Kapovich and M. Lustig, "The actions of $\operatorname{Out}\left(F_{k}\right)$ on the boundary of outer space and on the space of currents: Minimal sets and equivariant incompatibility", Ergodic Theory Dynam. Systems 27:3 (2007), 827-847. MR 2008h:20051 Zbl 1127.20025

[Kapovich and Lustig 2009] I. Kapovich and M. Lustig, "Geometric intersection number and analogues of the curve complex for free groups", Geom. Topol. 13:3 (2009), 1805-1833. MR 2010h: 20092 Zbl 1194.20046

[Kapovich and Lustig 2010a] I. Kapovich and M. Lustig, "Intersection form, laminations and currents on free groups", Geom. Funct. Anal. 19:5 (2010), 1426-1467. MR 2011g:20052 Zbl 1242.20052

[Kapovich and Lustig 2010b] I. Kapovich and M. Lustig, "Ping-pong and outer space", J. Topol. Anal. 2:2 (2010), 173-201. MR 2011d:20055 Zbl 1211.20027

[Kapovich and Nagnibeda 2007] I. Kapovich and T. Nagnibeda, "The Patterson-Sullivan embedding and minimal volume entropy for outer space", Geom. Funct. Anal. 17:4 (2007), 1201-1236. MR 2009c:20073 Zbl 1135.20031

[Kapovich and Nagnibeda 2010] I. Kapovich and T. Nagnibeda, "Geometric entropy of geodesic currents on free groups", pp. 149-175 in Dynamical numbers-interplay between dynamical systems and number theory, edited by S. Kolyada et al., Contemp. Math. 532, Amer. Math. Soc., Providence, RI, 2010. MR 2762139 Zbl 1216.20034

[Ladra et al. 2015] M. Ladra, P. V. Silva, and E. Ventura, "Bounding the gap between a free group (outer) automorphism and its inverse", Collectanea Mathematica (online publication February 2015).

[Levitt 2009] G. Levitt, "Counting growth types of automorphisms of free groups", Geom. Funct. Anal. 19:4 (2009), 1119-1146. MR 2011f:20068 Zbl 1196.20038

[Lyons 1994] R. Lyons, "Equivalence of boundary measures on covering trees of finite graphs", Ergodic Theory Dynam. Systems 14:3 (1994), 575-597. MR 95g:58132 Zbl 0821.58008

[Mann and Reynolds 2013] B. Mann and P. Reynolds, "Constructing non-uniquely ergodic arational trees", preprint, 2013. arXiv 1311.1771

[Martin 1995] R. Martin, Non-uniquely ergodic foliations of thin-type, measured currents and automorphisms of free groups, Ph.D. thesis, University of California, Los Angeles, 1995, Available at http://search.proquest.com/docview/304185823.

[Paulin 1989] F. Paulin, “The Gromov topology on R-trees”, Topology Appl. 32:3 (1989), 197-221. MR 90k:57015 Zbl 0675.20033

[Pollicott and Sharp 2014] M. Pollicott and R. Sharp, "A Weil-Petersson type metric on spaces of metric graphs”, Geom. Dedicata 172 (2014), 229-244. MR 3253781 Zbl 1301.30043

[Reynolds 2012] P. Reynolds, "Reducing systems for very small trees", preprint, 2012. arXiv 1211. 3378

[Sharp 2010] R. Sharp, "Distortion and entropy for automorphisms of free groups", Discrete Contin. Dyn. Syst. 26:1 (2010), 347-363. MR 2011e:37073 Zbl 1220.37018

[White 1991] T. P. White, The geometry of the outer space, Ph.D. thesis, University of California, Los Angeles, 1991, Available at http://search.proquest.com/docview/303915773.

Received August 26, 2014. Revised February 12, 2015. 
ILYA KAPOVICH

DEPARTMENT OF MATHEMATICS

UNIVERSITY OF ILLINOIS AT URBANA-CHAMPAIGN

1409 WEST GREEN STREET

URBANA, IL 61801

UNITED STATES

kapovich@math.uiuc.edu

MARTIN LUSTIG

Centre de Mathématiques et Informatique

AiX-MARSEILle Université

39, RUE F. Joliot CURIE

13453 MARSEILLE 13

FRANCE

martin.lustig@univ-amu.fr 


\title{
PACIFIC JOURNAL OF MATHEMATICS
}

\author{
msp.org/pjm
}

Founded in 1951 by E. F. Beckenbach (1906-1982) and F. Wolf (1904-1989)

\section{EDITORS}

Don Blasius (Managing Editor)

Department of Mathematics

University of California

Los Angeles, CA 90095-1555

blasius@math.ucla.edu

\author{
Paul Balmer \\ Department of Mathematics \\ University of California \\ Los Angeles, CA 90095-1555 \\ balmer@math.ucla.edu \\ Robert Finn \\ Department of Mathematics \\ Stanford University \\ Stanford, CA 94305-2125 \\ finn@math.stanford.edu \\ Sorin Popa \\ Department of Mathematics \\ University of California \\ Los Angeles, CA 90095-1555 \\ popa@math.ucla.edu
}

\author{
Vyjayanthi Chari \\ Department of Mathematics \\ University of California \\ Riverside, CA 92521-0135 \\ chari@math.ucr.edu \\ Kefeng Liu \\ Department of Mathematics \\ University of California \\ Los Angeles, CA 90095-1555 \\ liu@math.ucla.edu \\ Jie Qing \\ Department of Mathematics \\ University of California \\ Santa Cruz, CA 95064 \\ qing@ cats.ucsc.edu
}

\section{PRODUCTION}

Silvio Levy, Scientific Editor, production@msp.org

\section{SUPPORTING INSTITUTIONS}

ACADEMIA SINICA, TAIPEI

CALIFORNIA INST. OF TECHNOLOGY

INST. DE MATEMÁTICA PURA E APLICADA

KEIO UNIVERSITY

MATH. SCIENCES RESEARCH INSTITUTE

NEW MEXICO STATE UNIV.

OREGON STATE UNIV.

\author{
STANFORD UNIVERSITY \\ UNIV. OF BRITISH COLUMBIA \\ UNIV. OF CALIFORNIA, BERKELEY \\ UNIV. OF CALIFORNIA, DAVIS \\ UNIV. OF CALIFORNIA, LOS ANGELES \\ UNIV. OF CALIFORNIA, RIVERSIDE \\ UNIV. OF CALIFORNIA, SAN DIEGO \\ UNIV. OF CALIF., SANTA BARBARA
}

\author{
Daryl Cooper \\ Department of Mathematics \\ University of California \\ Santa Barbara, CA 93106-3080 \\ cooper@math.ucsb.edu \\ Jiang-Hua Lu \\ Department of Mathematics \\ The University of Hong Kong \\ Pokfulam Rd., Hong Kong \\ jhlu@maths.hku.hk \\ Paul Yang \\ Department of Mathematics \\ Princeton University \\ Princeton NJ 08544-1000 \\ yang@math.princeton.edu
}

These supporting institutions contribute to the cost of publication of this Journal, but they are not owners or publishers and have no responsibility for its contents or policies.

See inside back cover or msp.org/pjm for submission instructions.

The subscription price for 2015 is US \$420/year for the electronic version, and \$570/year for print and electronic.

Subscriptions, requests for back issues and changes of subscribers address should be sent to Pacific Journal of Mathematics, P.O. Box 4163, Berkeley, CA 94704-0163, U.S.A. The Pacific Journal of Mathematics is indexed by Mathematical Reviews, Zentralblatt MATH, PASCAL CNRS Index, Referativnyi Zhurnal, Current Mathematical Publications and Web of Knowledge (Science Citation Index).

The Pacific Journal of Mathematics (ISSN 0030-8730) at the University of California, c/o Department of Mathematics, 798 Evans Hall \#3840, Berkeley, CA 94720-3840, is published twelve times a year. Periodical rate postage paid at Berkeley, CA 94704, and additional mailing offices. POSTMASTER: send address changes to Pacific Journal of Mathematics, P.O. Box 4163, Berkeley, CA 94704-0163.

PJM peer review and production are managed by EditFLOW ${ }^{\circledR}$ from Mathematical Sciences Publishers.

\section{PUBLISHED BY}

\section{mathematical sciences publishers \\ nonprofit scientific publishing}

http://msp.org/

(C) 2015 Mathematical Sciences Publishers 


\section{PACIFIC JOURNAL OF MATHEMATICS}

Volume $277 \quad$ No. $2 \quad$ October 2015

The Borel-Weil theorem for reductive Lie groups

JosÉ ARAUJO and TIM BRATTEN

A curvature flow unifying symplectic curvature flow and pluriclosed

287 flow

SONG DAI

Representations of knot groups into $\mathrm{SL}_{n}(\mathbb{C})$ and twisted Alexander polynomials

Michael HeUsener and JoAn PORTi

Approximations by maximal Cohen-Macaulay modules

HENRIK HOLM

Patterson-Sullivan currents, generic stretching factors and the asymmetric Lipschitz metric for outer space

ILYA KAPOVICH and MARTIN LUSTIG

On recurrence over subsets and weak mixing

JiAn Li, Piotr Oprocha and GUOHUA Zhang

Représentations de Steinberg modulo $p$ pour un groupe réductif sur un 425 corps local

TONY LY

Calculating two-strand jellyfish relations

DAVID PENNEYS and EMILY PETERS 